This article has been downloaded from IOPscience. Please scroll down to see the full text article. (http://iopscience.iop.org/1126-6708/2007/10/092)

More related content is available

Download details:

IP Address: 137.138.125.160

The article was downloaded on 12/12/2007 at 14:15

Please note that terms and conditions apply. 


\title{
WMAP-compliant benchmark surfaces for MSSM Higgs bosons
}

\author{
John Ellis, ${ }^{a}$ Thomas Hahn, ${ }^{b}$ Sven Heinemeyer, ${ }^{c}$ Keith A. Olive $^{d}$ and Georg Weiglein ${ }^{e}$ \\ ${ }^{a}$ TH Division, Physics Department, CERN, Geneva, Switzerland \\ ${ }^{b}$ Max-Planck-Institut für Physik, \\ Föhringer Ring 6, D-80805 Munich, Germany \\ ${ }^{c}$ Instituto de Fisica de Cantabria (CSIC-UC), Santander, Spain \\ ${ }^{d}$ William I. Fine Theoretical Physics Institute, \\ University of Minnesota, Minneapolis, MN 55455, U.S.A. \\ e IPPP, University of Durham, Durham DH1 3LE, U.K. \\ E-mail: John.Ellis@cern.ch, hahn@feynarts.de, Sven.Heinemeyer@cern.ch, \\ OLIVE@mnhep.hep.umn.edu, Georg.Weiglein@durham.ac.uk
}

ABSTRACT: We explore 'benchmark surfaces' suitable for studying the phenomenology of Higgs bosons in the minimal supersymmetric extension of the Standard Model (MSSM), which are chosen so that the supersymmetric relic density is generally compatible with the range of cold dark matter density preferred by WMAP and other observations. These benchmark surfaces are specified assuming that gaugino masses $m_{1 / 2}$, soft trilinear supersymmetry-breaking parameters $A_{0}$ and the soft supersymmetry-breaking contributions $m_{0}$ to the squark and slepton masses are universal, but not those associated with the Higgs multiplets (the NUHM framework). The benchmark surfaces may be presented as $\left(M_{A}, \tan \beta\right)$ planes with fixed or systematically varying values of the other NUHM parameters, such as $m_{0}, m_{1 / 2}, A_{0}$ and the Higgs mixing parameter $\mu$. We discuss the prospects for probing experimentally these benchmark surfaces at the Tevatron collider, the LHC, the ILC, in $B$ physics and in direct dark-matter detection experiments. An appendix documents developments in the FeynHiggs code that enable the user to explore for her/himself the WMAP-compliant benchmark surfaces.

Keywords: Supersymmetry Phenomenology, Higgs Physics, Supersymmetric Standard Model, GUT. 


\section{Contents}

1. Introduction 1

2. Specification of the benchmark surfaces

3. Electroweak precision observables 8

4. Tevatron phenomenology 9

5. LHC phenomenology 10

6. ILC phenomenology 16

7. $B$ physics 19

8. Direct detection of supersymmetric dark matter 19

9. Conclusions 22

A. Evaluation of benchmark surfaces with FeynHiggs 23

A.1 Fortran use 24

A.1.1 Declaration 24

A.1.2 Initializing a record 24

A.1.3 Looping over a record/setting the FeynHiggs input 26

A.1.4 Associating a record with a table 26

A.2 Mathematica use 27

A.3 Examples 28

A.3.1 Command-line mode with parameter table 28

A.3.2 Using a record with table in Fortran 29

A.3.3 Using a record with table in Mathematica 30

\section{Introduction}

Some of the best prospects for probing the minimal supersymmetric extension of the Standard Model (MSSM) [1, 2] might be offered by searches for the bosons appearing in its extended Higgs sector. It may be challenging to distinguish between the lightest MSSM Higgs boson and a Standard Model (SM) Higgs boson with the same mass, and searches for MSSM Higgs bosons are, in many ways, complementary to searches for supersymmetric particles as avenues to establish the existence of physics beyond the SM. 
Searches at the Tevatron collider are closing in on the possible existence of an SM-like Higgs boson over a limited range of low masses [3-5], and are also starting to encroach significantly on the options for heavier MSSM Higgs bosons, particularly at large $\tan \beta$ [610]. Studies have shown that experiments at the LHC will be able to establish the existence or otherwise of an SM-like Higgs boson over all its possible mass range, and also explore many options for the heavier MSSM Higgs bosons [11-14]. On the other hand, the LHC might well be unable to distinguish between the lightest MSSM Higgs boson and an SM Higgs boson of the same mass. The ILC would have better chances of making such a distinction [15-22], and might also be able to produce the other MSSM Higgs bosons if they are not too heavy [15-19]. CLIC would also be able to study a light SM-like Higgs boson, as well as extend the search for MSSM Higgs bosons to much higher masses [23]. Searches for new phenomena in $B$ physics, including rare decays such as $b \rightarrow s \gamma, B_{s} \rightarrow \mu^{+} \mu^{-}$ and $B_{u} \rightarrow \tau \nu$, also have good potential to explore the MSSM Higgs sector and, at least in some specific MSSM scenarios, electroweak precision observables (EWPO) may also provide interesting constraints [24, 25]. In parallel to these accelerator searches for MSSM Higgs bosons and their effects, non-accelerator searches for supersymmetric dark matter [26, 27] will also be able to explore significant regions of the MSSM Higgs parameter space [28[30], since the exchanges of massive MSSM Higgs bosons have significant impacts on dark matter scattering cross sections.

In order to correlate the implications of searches at hadron colliders and linear colliders, in $B$ physics, in dark matter searches and elsewhere, it is desirable to define MSSM Higgs benchmark scenarios that are suitable for comparing and assessing the relative scopes of different search strategies, see, e.g., refs. [31-38].

Since the MSSM Higgs sector is governed by the two parameters $M_{A}$ (or $M_{H^{ \pm}}$) and $\tan \beta$ at lowest order, aspects of MSSM Higgs-boson phenomenology such as current exclusion bounds and the sensitivities of future searches are usually displayed in terms of these two parameters. The other MSSM parameters enter via higher-order corrections, and are conventionally fixed according to certain benchmark definitions 31-34. The benchmark scenarios commonly used in the literature encompass a range of different possibilities for the amount of mixing between the scalar top quarks, which have significant implications for MSSM Higgs phenomenology, and also include the possibility of radiatively-induced $\mathcal{C P}$ violation. The best-known example is the so-called " $m_{h}^{\max }$ scenario" 31-33, which allows the search for the light $\mathcal{C P}$-even Higgs boson to be translated into conservative bounds on $\tan \beta$ for fixed values of the top-quark mass and the scale of the supersymmetric particles [39]. The existing benchmark scenarios designed for the MSSM Higgs sector are formulated entirely in terms of low-scale parameters, i.e., they are not related to any particular SUSY-breaking scheme and make no provision for a possible unification of the SUSY-breaking parameters at some high mass scale, as occurs in generic supergravity and string scenarios.

In applications of the existing benchmark scenarios for the MSSM Higgs sector [3134], one is normally concerned only with the phenomenology of the Higgs sector itself. Besides the direct searches for supersymmetric particles, other constraints arising from EWPO, B-physics observables (BPO) and the possible supersymmetric origin of the as- 
trophysical cold dark matter $(\mathrm{CDM})$ are not usually taken into account. This may be motivated by the fact that the additional constraints from EWPO, BPO and CDM can depend sensitively on soft-supersymmetry breaking parameters that otherwise have minor impacts on Higgs phenomenology. For example, the presence of small flavour-mixing terms in the MSSM Lagrangian would severely affect the predictions for the BPO while leaving Higgs phenomenology essentially unchanged (see also ref. [36] for a discussion of this issue).

In this paper we follow a different approach and adopt specific universality assumptions about the soft SUSY-breaking parameters, restricting our analysis of the MSSM to a wellmotivated subspace of manageable dimensionality. It is frequently assumed that the scalar masses $m_{0}$ are universal at some high unification scale, as are the gaugino masses $m_{1 / 2}$ and the trilinear parameters $A_{0}$, a framework known as the constrained MSSM (CMSSM). In such a scenario, the heavier MSSM Higgs boson masses are fixed in terms of the input parameters and $\tan \beta$, so that $M_{A}$ is not an independent parameter, and consequently this scenario is too restrictive for our purposes. However, there is no good phenomenological or theoretical reason why the soft supersymmetry-breaking contributions to the Higgs masses should not be non-universal, a scenario termed the NUHM [40-42]. Within the NUHM, $M_{A}$ and $\mu$ can be treated as free parameters for any specified values of $m_{0}, m_{1 / 2}, A_{0}$ and $\tan \beta$, so that this scenario provides a suitable framework for studying the phenomenology of the MSSM Higgs sector. Since the low-scale parameters in this scenario are derived from a small set of input quantities in a meaningful way, it is of interest to take into account other experimental constraints.

The main purpose of this paper is to explore new benchmark surfaces for MSSM Higgs phenomenology that are compatible with the cosmological density of cold dark matter inferred from a combination of WMAP and other observations [43]. While in the CMSSM only narrow strips in $\left(m_{1 / 2}, m_{0}\right)$ planes are compatible with WMAP et al. 444, 45] for given values of $A_{0}$ and $\tan \beta$, the NUHM offers the attractive possibility to specify $\left(M_{A}, \tan \beta\right)$ planes such that essentially the whole plane is allowed by the constraints from WMAP and other observations [25]. This is done assuming that $R$ parity is conserved, that the lightest supersymmetric particle (LSP) is the lightest neutralino $\tilde{\chi}_{1}^{0}$, and that it furnishes most of the cold dark matter required [46]. As we discuss in more detail below, compatibility with WMAP et al. cannot be maintained while keeping all the other NUHM parameters fixed. Accordingly, we discuss two examples of WMAP-compliant benchmark surfaces that are specified for fixed $m_{0}, \mu$ and $A_{0}=0$ but varying $m_{1 / 2}$, and two surfaces that are specified for fixed $m_{1 / 2}, m_{0}$ and $A_{0}=0$ but varying $\mu$. For the first two benchmark surfaces, a simple linear relation between $m_{1 / 2}$ and $M_{A}$ is imposed as the $\left(M_{A}, \tan \beta\right)$ plane is scanned, whereas for the other two surfaces $\mu$ is varied through a relatively narrow range.

Following the specifications of these NUHM benchmark surfaces, we then explore the possibilities for studies of the MSSM Higgs bosons and other supersymmetric signatures across these $\left(M_{A}, \tan \beta\right)$ planes. We consider the electroweak precision observables, principally $a_{\mu} \equiv \frac{1}{2}(g-2)_{\mu}$ and $M_{h}$, prospects for the search for $H / A \rightarrow \tau^{+} \tau^{-}$at the Tevatron, prospects at the LHC - including searches for $h \rightarrow \gamma \gamma$ and $\tau^{+} \tau^{-}, H / A \rightarrow \tau^{+} \tau^{-}$and $H^{ \pm} \rightarrow \tau^{ \pm} \nu$, and measurements of the ratio of $h \rightarrow \tau^{+} \tau^{-}$and $W W^{*}$ branching ratios, prospects at the ILC - including ways of distinguishing between the light MSSM $h$ bo- 
son and an SM Higgs boson of the same mass by measuring (ratios of) branching ratios, prospects in $B$ physics - including $B_{s} \rightarrow \mu^{+} \mu^{-}, b \rightarrow s \gamma$ and $B_{u} \rightarrow \tau \nu$, and the direct detection of supersymmetric cold dark matter. In an appendix we introduce developments in the FeynHiggs code that enable the user to explore for her/himself the WMAP-compliant benchmark surfaces. These include the concept of a FeynHiggs record, a new data type that captures the entire content of a parameter file in the native format of FeynHiggs.

\section{Specification of the benchmark surfaces}

As an introduction to the specification of the benchmark surfaces in the NUHM, we first consider a generic $\left(M_{A}, \tan \beta\right)$ plane for fixed $m_{1 / 2}, m_{0}, A_{0}$ and $\mu$, adapted from ref. 47. As we see in figure 1(a), in the $\left(M_{A}, \tan \beta\right)$ plane for $m_{1 / 2}=600 \mathrm{GeV}, m_{0}=800 \mathrm{GeV}$, $\mu=1000 \mathrm{GeV}$ and $A_{0}=0$, the relic LSP density satisfies the WMAP constraint only in narrow, near-vertical (pale blue) shaded strips crossing the plane. These lie to either side of the vertical (purple) line where $m_{\tilde{\chi}_{1}^{0}}=M_{A} / 2$. Within the narrow unshaded strip straddling this line, the relic density is suppressed by rapid direct-channel annihilations to a value below the lower limit of the range for the cold dark matter density indicated by WMAP et al. This strip would be acceptable for cosmology if there were some additional component of cold dark matter. Outside the shaded WMAP-compatible strips, at both larger and smaller values of $M_{A}$, the relic LSP density is too high, and these regions are unacceptable. ${ }^{1}$

It is clear from this example that one may arrange for the relic LSP density to remain within the preferred WMAP range over (essentially) the entire $\left(M_{A}, \tan \beta\right)$ plane if one adjusts $m_{1 / 2}$ continuously as a function of $M_{A}$ so as to remain within one of the narrow WMAP strips as $M_{A}$ increases. Accordingly, we study a benchmark $\left(M_{A}, \tan \beta\right)$ plane P1 with the same values of $m_{0}=800 \mathrm{GeV}, \mu=1000 \mathrm{GeV}$ and $A_{0}=0$, but with varying $m_{1 / 2} \sim \frac{9}{8} M_{A}$. Since we evaluate observables using a discrete sampling of the NUHM parameter space, we consider values of $m_{1 / 2}$ lying within the small range of this central value:

$$
\frac{9}{8} M_{A}-12.5 \mathrm{GeV} \leq m_{1 / 2} \leq \frac{9}{8} M_{A}+37.5 \mathrm{GeV} .
$$

The observables that we study do not vary significantly as $m_{1 / 2}$ is varied across this range. Specifically, we use the $m_{1 / 2}$ that gives the value of the cold dark matter density that is closest to the central value within the allowed range, $0.0882<\Omega_{\mathrm{CDM}} h^{2}<0.1204$ 43] (see below).

Previous analyses of the CMSSM indicated that values of $m_{1 / 2}$ and $m_{0}$ below $1 \mathrm{TeV}$ are preferred, in particular by the EWPO [48, 49, 25] (see also ref. [50]). Accordingly, we study also a benchmark $\left(M_{A}, \tan \beta\right)$ plane P2 with the fixed values $m_{0}=300 \mathrm{GeV}$, $\mu=800 \mathrm{GeV}$ and $A_{0}=0$, with $m_{1 / 2} \sim 1.2 M_{A}$ again varying continuously across the plane

\footnotetext{
${ }^{1}$ We note in passing that the LEP lower limit on $M_{h}$ excludes a strip of this plane at low $M_{A}$ and/or $\tan \beta$ indicated by the dash-dotted (red) line, that $a_{\mu}$ (pink shading) prefers relatively large $\tan \beta>36$, that $b \rightarrow s \gamma$ excludes a (green shaded) region at low $M_{A}$ and $\tan \beta$, and that the other BPO disfavour a region at low $M_{A}$ and high $\tan \beta$ (not shown).
} 

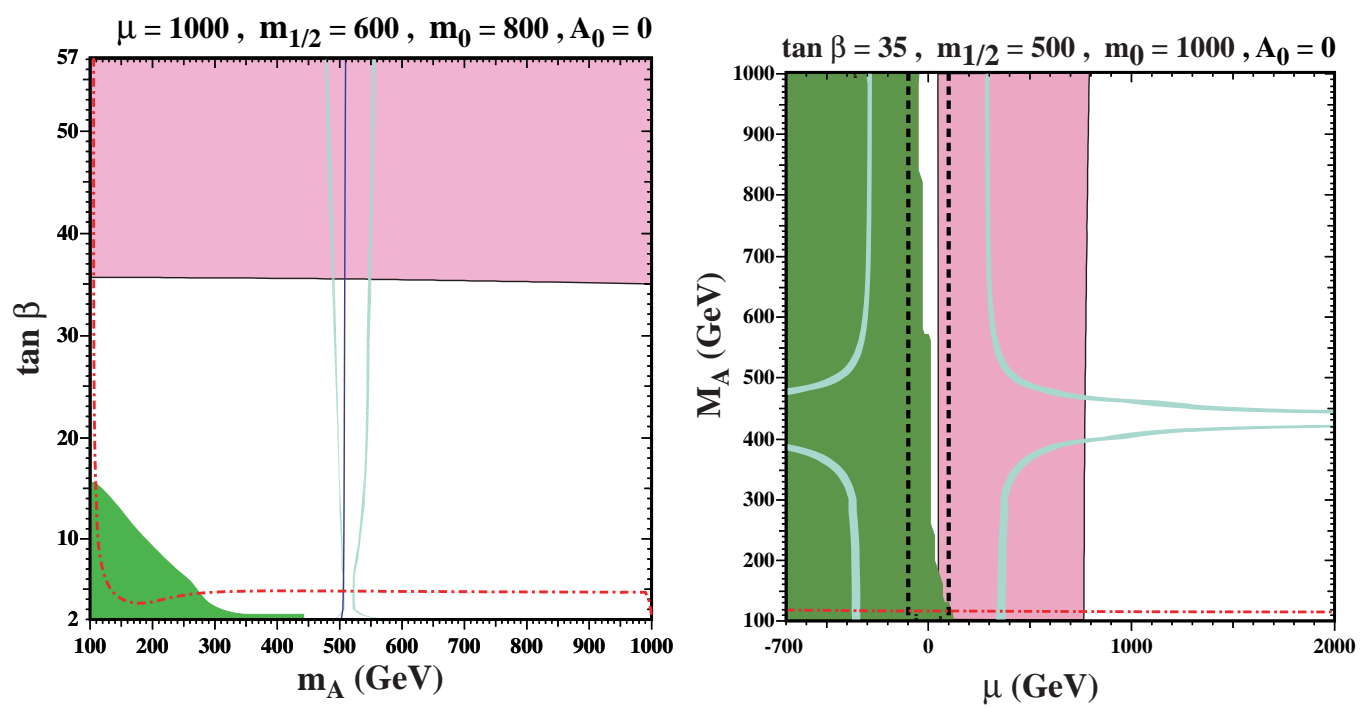

Figure 1: Sample NUHM parameter planes with two parameters varied and the other four fixed, adapted from refs. [47, 51. The left plot displays a $\left(M_{A}, \tan \beta\right)$ plane with $m_{1 / 2}=600 \mathrm{GeV}$, $m_{0}=800 \mathrm{GeV}, \mu=1000 \mathrm{GeV}$ and $A_{0}=0$. The range of cold dark matter density preferred by WMAP and other observations is attained in two narrow (pale blue) strips, one on either side of the vertical solid (blue) line where $m_{\tilde{\chi}_{1}^{0}}=M_{A} / 2$. The dark (green) shaded region at low $M_{A}$ and low $\tan \beta$ is excluded by $b \rightarrow s \gamma$, and the medium (pink) shaded region at $\tan \beta>36$ is favoured by $a_{\mu}$. The region below the (red) dot-dashed line is excluded by the LEP bounds on $M_{h}$. The right plot displays a $\left(\mu, M_{A}\right)$ plane with $m_{1 / 2}=500 \mathrm{GeV}, m_{0}=1000 \mathrm{GeV}, \tan \beta=35$ and $A_{0}=0$. Here the WMAP range of cold dark matter density is attained in two narrow strips at roughly constant positive and negative values of $\mu$, which are swept apart by rapid annihilation when $M_{A} \sim 2 m_{\tilde{\chi}_{1}^{0}}$. The dark (green) shaded region at $\mu<0$ is excluded by $b \rightarrow s \gamma$, and the $0<\mu<760 \mathrm{GeV}$ strip (pink shading) is favoured by $a_{\mu}$. The region below the (red) dot-dashed line again is excluded by the LEP bounds on $M_{h}$, and the region between the vertical (black) dashed lines has $m_{\tilde{\chi}_{1}^{ \pm}}<104 \mathrm{GeV}$.

so as to maintain the WMAP relationship with $M_{A}$. As before, because of our discrete sampling of the NUHM parameter space, we consider values of $m_{1 / 2}$ lying within a small range of this central value:

$$
1.2 M_{A}-40 \mathrm{GeV} \leq m_{1 / 2} \leq 1.2 M_{A}+40 \mathrm{GeV} .
$$

Again, the observables that we study do not vary significantly as $m_{1 / 2}$ is varied across this range.

More examples could be chosen with different fixed values of $m_{0}, \mu$ and $A_{0}$ but, as long as $m_{1 / 2}$ is the parameter being varied to keep the LSP density within the WMAP range, a similar relationship between $m_{1 / 2}$ and $M_{A}$ will always apply. The only flexibility in the choice of $m_{1 / 2}$ is whether one wishes to stay within the left or right near-vertical shaded strip. However, the corresponding values of $m_{1 / 2}$ do not differ greatly, and neither do the corresponding phenomenological signatures, though the lightest Higgs boson mass can be somewhat sensitive to this choice. The values of $m_{0}$ and (to a lesser extent) $\mu$ have far more 
impact on the phenomenology, and the benchmark choices we have made: $m_{0}=800 \mathrm{GeV}$ for $\mathbf{P 1}$ and $m_{0}=300 \mathrm{GeV}$ for $\mathbf{P 2}$, provide significant and interesting differences worthy of examination.

We also study two other $\left(M_{A}, \tan \beta\right)$ planes, whose motivation can be gained from examination of the $\left(\mu, M_{A}\right)$ plane shown in figure 1 $(\mathrm{b})$, which is adapted from ref. [51]. We see that, for a fixed choice of values of $m_{1 / 2}=500 \mathrm{GeV}, m_{0}=1000 \mathrm{GeV}$ and $A_{0}=0$, there is a narrow strip of values of $\mu \sim 300 \rightarrow 350 \mathrm{GeV}$ where the relic density lies within the WMAP range for almost all values of $M_{A}$. The exception is a narrow strip centred on $M_{A} \sim 430 \mathrm{GeV}$, namely the rapid-annihilation funnel where $m_{\tilde{\chi}_{1}^{0}} \sim M_{A} / 2$, which would be acceptable if there is some other source of cold dark matter. This funnel is narrower (wider) for smaller (larger) values of $\tan \beta$, but its location in $\mu$ does not vary much as a function of $\tan \beta .^{2}$

Motivated by this example, we explore two benchmark surfaces with different fixed values of $m_{1 / 2}$ and $m_{0}$, and $\mu$ varying within a restricted range chosen to maintain the LSP density within or below the WMAP range. The first example of such a benchmark plane, P3, has fixed $m_{1 / 2}=500 \mathrm{GeV}, m_{0}=1000 \mathrm{GeV}$ and $A_{0}=0$, with $\mu$ in the range

$$
\mu=250-400 \mathrm{GeV}
$$

In the following, we evaluate observables for a discrete sampling of $\mu$ values within this range. Since the corresponding variation of the particle mass spectrum is quite small, the impact of the variation of $\mu$ on the observables discussed below is negligible.

The other example of such a benchmark plane, $\mathbf{P} \mathbf{4}$, has fixed $m_{1 / 2}=300 \mathrm{GeV}, m_{0}=$ $300 \mathrm{GeV}$ and $A_{0}=0$, with $\mu$ in the range

$$
\mu=200-350 \mathrm{GeV} .
$$

As in the previous case, the LSP density lies within the WMAP range except for a small range of $M_{A} \sim 2 m_{\tilde{\chi}_{1}^{0}}$ where the density is below the preferred range. However, again this is acceptable if there is some other component of cold dark matter. The parameter choices for this and the other NUHM benchmark surfaces are summarized in table 1 . $^{3}$

A likelihood analysis of these four NUHM benchmark surfaces, including the EWPO $M_{W}, \sin ^{2} \theta_{\text {eff }}, \Gamma_{Z},(g-2)_{\mu}$ and $M_{h}$ and the $\operatorname{BPO} \operatorname{BR}(b \rightarrow s \gamma), \operatorname{BR}\left(B_{s} \rightarrow \mu^{+} \mu^{-}\right)$, $\operatorname{BR}\left(B_{u} \rightarrow \tau \nu_{\tau}\right)$ and $\Delta M_{B_{s}}$ was performed recently in ref. [25]. The lowest $\chi^{2}$ value in each plane, denoted as $\chi_{\min }^{2}$, is shown in the rightmost column of table 1, corresponding to the points labeled as the best fits in the plots below. We display in each of the following figures the locations of these best-fit points by a (red) cross and the $\Delta \chi^{2}=2.30$ and 4.61 contours around the best-fit points in the $\left(M_{A}, \tan \beta\right)$ planes for each of these benchmark surfaces. These contours would correspond to the $68 \%$ and $95 \%$ C.L. contours in

\footnotetext{
${ }^{2}$ We note in passing that the LEP lower limit on $M_{h}$ excludes a strip of this plane at low $M_{A}$ indicated by the (red) dash-dotted line, and the LEP lower limit on the chargino mass excludes values of $\mu$ between the two vertical (black) dashed lines.

${ }^{3} \mathrm{~A}$ minor change in the best-fit point and the $\chi_{\min }^{2}$ ocurred for the $\mathbf{P 2}$ scenario in comparison with ref. 25] due to a slightly different choice of the $m_{1 / 2}$ values.
} 


\begin{tabular}{|c|c|c|c|c|c|}
\cline { 2 - 6 } \multicolumn{1}{c|}{} & $m_{1 / 2}$ & $m_{0}$ & $A_{0}$ & $\mu$ & $\chi_{\min }^{2}$ \\
\hline P1 & $\sim \frac{9}{8} M_{A}$ & 800 & 0 & 1000 & 7.1 \\
\hline P2 & $\sim 1.2 M_{A}$ & 300 & 0 & 800 & 3.1 \\
\hline P3 & 500 & 1000 & 0 & $250 \ldots 400$ & 7.4 \\
\hline P4 & 300 & 300 & 0 & $200 \ldots 350$ & 5.6 \\
\hline
\end{tabular}

Table 1: The four NUHM benchmark surfaces are specified by the above fixed and varying parameters, allowing $M_{A}$ and $\tan \beta$ to vary freely. All mass parameters are in $\mathrm{GeV}$. The rightmost column shows the minimum $\chi^{2}$ value found in each plane at the points labelled as the best fits in the plots.

the $\left(M_{A}, \tan \beta\right)$ planes if the overall likelihood distribution, $\mathcal{L} \propto e^{-\chi^{2} / 2}$, were Gaussian. This is clearly only approximately true, but these contours nevertheless give interesting indications on the regions in the $\left(M_{A}, \tan \beta\right)$ planes that are currently preferred. The varied parameter in each scenario (i.e. $m_{1 / 2}$ in $\mathbf{P 1}, \mathbf{P 2}$ and $\mu$ in $\mathbf{P 3}, \mathbf{P} 4$ ) is chosen such that the cold dark matter density is closest to the central value within the allowed range, $0.0882<\Omega_{\mathrm{CDM}} h^{2}<0.1204$ 43.

On surfaces $\mathbf{P} 1$ and $\mathbf{P 2}$, where $m_{1 / 2}$ scales with $M_{A}$ so as to remain in the funnel region, much of the mass spectrum scales with $M_{A}$. Specifically, the lightest neutralino and chargino masses simply scale in direct proportion to $M_{A}$ for these surfaces. The light squark masses and stau masses also scale with $m_{1 / 2}$ (and hence $M_{A}$ ), though the latter are also slightly dependent on $\tan \beta$ as well. In the range $M_{A} \leq 1 \mathrm{TeV}$ displayed in these planes, the light squark masses range up to $\sim 2.3 \mathrm{TeV}$ for surface $\mathbf{P} \mathbf{1}$, within reach of the LHC. However, because of the relatively large values of $m_{0}$, the light squarks are beyond the current reach of the Tevatron collider even at low $M_{A}$ (and hence $m_{1 / 2}$ ). For P2, the light squark masses range up to $\sim 1.7 \mathrm{TeV}$.

Turning to surfaces P3 and P4, because they have fixed values of $m_{1 / 2}$ and $m_{0}$, there are very small variations in the sparticle mass spectra across these planes. For example, the lightest neutralino and chargino masses are determined primarily by $m_{1 / 2}$, and so they both take almost constant values on the benchmark surfaces. Similarly, the light squark masses are determined by a combination of $m_{1 / 2}$ and $m_{0}$ and show little dependence on either $M_{A}$ or $\tan \beta$. On the other hand, the lightest stau mass has a slight dependence on $\tan \beta$, due to the variable splitting of the third-generation sparticle masses. These mass splittings increase at large $\tan \beta$, leading to smaller stau masses.

We display in each plane the region excluded (black shaded) at the $95 \%$ C.L. by the LEP Higgs searches in the channel $e^{+} e^{-} \rightarrow Z^{*} \rightarrow Z h, H$ [53, 52]. For a SM-like Higgs boson we use a bound of $M_{h}>113 \mathrm{GeV}$. The difference from the nominal LEP mass limit allows for the estimated theoretical uncertainty in the calculation of $M_{h}$ for specific values of the input MSSM parameters [54]. In the region of small $M_{A}$ and large $\tan \beta$, where the coupling of the light $\mathcal{C} \mathcal{P}$-even Higgs boson to gauge bosons is suppressed, the bound on $M_{h}$ is reduced to $M_{h}>91 \mathrm{GeV}$ [52]. 


\section{Electroweak precision observables}

In this section we summarize key predictions for electroweak precision observables (EWPO) over the four benchmark surfaces. In ref. [25] it was shown that $M_{W}, \sin ^{2} \theta_{\text {eff }}$ and $\Gamma_{Z}$ agree within $\sim 1 \sigma$ with the current experimental value over all the benchmark surfaces. Since their variations are relatively small, we do not display these observables in this paper, though they are included in the overall $\chi^{2}$ function. Here we focus on two other EWPO, namely the mass of the lightest Higgs boson, $M_{h}$, and the anomalous magnetic moment of the muon, $a_{\mu} \equiv \frac{1}{2}(g-2)_{\mu}$.

The evaluation of $M_{h}$ is performed using FeynHiggs [55, 56, 54, 57]. In figure 2 we show the contours for $M_{h}=113,114,115,116,117,118$ and $120 \mathrm{GeV}$. As discussed in the previous section, the boundary of the region excluded by the LEP searches for the lightest MSSM Higgs boson does not coincide with the nominal limit $M_{h}=114.4 \mathrm{GeV}$ on the mass of a Standard Model Higgs boson. Nevertheless, it can be seen in figure 2 that the $\Delta \chi^{2}=2.30$ and 4.61 contours are highly correlated with the $M_{h}$ contours at low values of $M_{A}$ and $\tan \beta$. This is a consequence of the fact that the full likelihood information from the LEP Higgs exclusion limit (as well as the theoretical uncertainty) is incorporated into the overall $\chi^{2}$ function (see ref. [25]). Note that for the plane P4 (and to a lesser extent P3) the maximum value for the Higgs mass is limited by the relatively low value of $m_{1 / 2}$.

Concerning $a_{\mu}$, we recall that, according to a recent evaluation of the Standard Model contribution based on low-energy $e^{+} e^{-}$data, there is a discrepancy with the experimental measurement by the E821 Collaboration [62, 63]. It would be premature to regard this deviation as solid evidence for new physics. However, within the SUSY framework we explore here, this discrepancy does impose a significant constraint on the parameter space, and makes an important contribution to the global $\chi^{2}$ function whose contours are shown in figure 2. Our evaluation of $a_{\mu}$ is based on refs. [58-61], which yields [64, 65]:

$$
a_{\mu}^{\text {exp }}-a_{\mu}^{\text {theo }}=(27.5 \pm 8.4) \times 10^{-10}
$$

equivalent to a $3.3-\sigma$ effect. ${ }^{4}$ In figure 2 we show the contours $\Delta a_{\mu}=10.7,19.1,35.9,44.3 \times$ $10^{-10}$ for the net supersymmetric contribution to $a_{\mu}$.

In the case of surface $\mathbf{P} 1$, we see that the best-fit point corresponds to $M_{h} \sim 118 \mathrm{GeV}$ and $\Delta a_{\mu} \sim 10.7 \times 10^{-10}$. In most of the displayed region of the surface that is favoured at the global $\Delta \chi^{2}<4.61$ level, $\Delta a_{\mu}$ is considerably lower than the range favoured in eq. (3.1). In the case of surface P2, the best-fit point has $M_{h} \sim 118 \mathrm{GeV}$, and $\Delta a_{\mu}$ is within the 1- $\sigma$ range given by eq. (3.1). In the case of surface P3, the best-fit point has $M_{h}>118 \mathrm{GeV}$ and again a low value of $\Delta a_{\mu}$. Finally, the best-fit point in surface $\mathbf{P} 4$ has $M_{h} \sim 115 \mathrm{GeV}$ and an excellent value of $\Delta a_{\mu}$, according to eq. (3.1). The fact that the best-fit points do not always have favoured values of $\Delta a_{\mu}$ reflects the importance of other precision observables, notably the BPO discussed later.

\footnotetext{
${ }^{4}$ Three other recent evaluations yield slightly different numbers 66 69, but similar discrepancies with the SM prediction.
} 

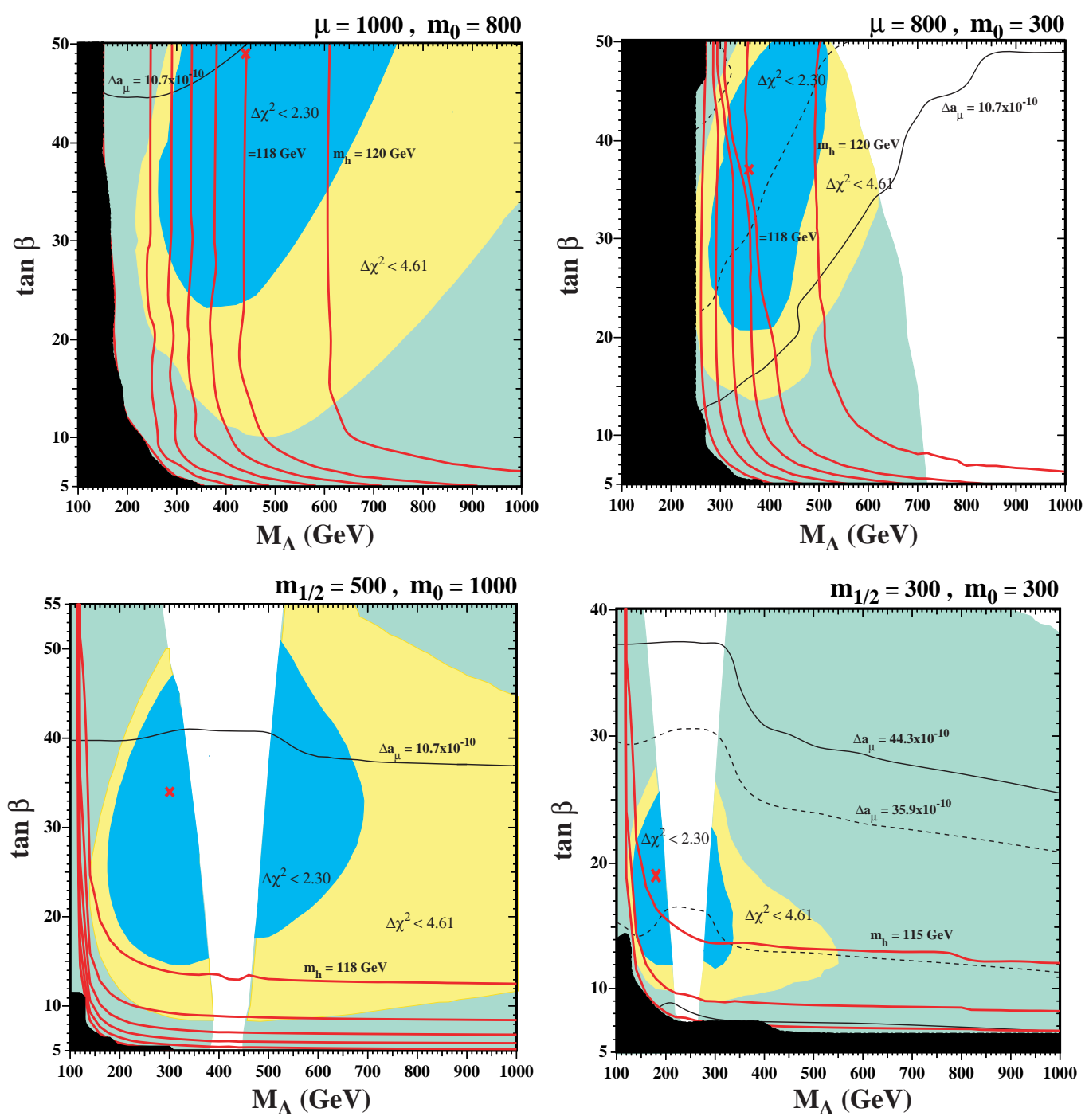

Figure 2: The $\left(M_{A}, \tan \beta\right)$ planes for the NUHM benchmark surfaces (a) P1, (b) P2, (c) P3 and (d) P4, displaying the contours of $\Delta \chi^{2}$ found in a recent global fit to EWPO and BPO [25]. All surfaces have $A_{0}=0$. We also display individually the contours of $M_{h}$ found using FeynHiggs 55 , 56, 54, 57] and the contours of $a_{\mu}$ found using refs. [58-61]. The 1(2)- $\sigma$ range for $a_{\mu}$ is demarcated by dashed (solid) lines. The dark shaded (black) region corresponds to the parameter region that is excluded by the LEP Higgs searches in the channel $e^{+} e^{-} \rightarrow Z^{*} \rightarrow Z h, H$ [53, 52].

\section{Tevatron phenomenology}

We first consider how experiments at the Tevatron collider in the next years could probe the benchmark surfaces $\mathbf{P 1}, \mathbf{P 2}, \mathbf{P 3}$ and $\mathbf{P 4}$. We consider one possible Tevatron signature for the MSSM Higgs sector, namely $H / A \rightarrow \tau^{+} \tau^{-}$, for which expectations are evaluated using the results from ref. [70]. They are based on the expectation of a $30 \%$ improvement in the sensitivity with respect to ref. [6]. We see in figure 3 that, at the Tevatron with $2(4$, 
8) $\mathrm{fb}^{-1}$ of integrated and analyzed luminosity per experiment, ${ }^{5}$ the channel $H / A \rightarrow \tau^{+} \tau^{-}$ would provide a $95 \%$ C.L. exclusion sensitivity to $\tan \beta \sim 35(30,25)$ when $M_{A} \sim 200 \mathrm{GeV}$, and the sensitivity decreases slowly (rapidly) at smaller (larger) $M_{A}$. In the case of the benchmark surface $\mathbf{P 1}, 8 \mathrm{fb}^{-1}$ would start accessing the region with $\Delta \chi^{2}<4.61$. For $\mathbf{P 2}$, however, the area accessible to the Tevatron is not visible in the figure since it is completely covered by the excluded region from the LEP Higgs searches. The region $\Delta \chi^{2}<4.61$ could be accessed already with $2 \mathrm{fb}^{-1}$ in case $\mathbf{P 3}$, and $8 \mathrm{fb}^{-1}$ would give access to the region with $\Delta \chi^{2}<2.30$. However, even the $\Delta \chi^{2}<4.61$ region of the $\mathbf{P} 4$ surface would be inaccessible with $8 \mathrm{fb}^{-1}$.

We note that the CDF Collaboration has recently reported $\mathrm{a} \sim 2-\sigma$ excess of candidate $H / A \rightarrow \tau^{+} \tau^{-}$events [9], which would correspond to $M_{A} \sim 160 \mathrm{GeV}$ and $\tan \beta>45$. As discussed in ref. [30], taking into account all the available experimental constraints, this possible excess could be accommodated within the NUHM only for rather different values of the parameters from those considered in the benchmark scenarios, namely $m_{1 / 2} \sim 650 \mathrm{GeV}$, $m_{0} \sim 1000 \mathrm{GeV}, A_{0} \sim-1900 \mathrm{GeV}, \mu \sim 385 \mathrm{GeV}$. A likelihood analysis yields values of $\chi^{2} \sim 9-10$, somewhat higher than the values for the benchmark surfaces. Within the four benchmark scenarios here, the precision observables are not in good agreement with low $M_{A}$ and large $\tan \beta$, reflecting the fact that the points with $M_{A} \sim 160 \mathrm{GeV}$ and $\tan \beta>45$ lie well outside the regions with $\Delta \chi^{2}<4.61$ on all of these benchmark surfaces.

\section{LHC phenomenology}

In this section we present and compare the sensitivities of various LHC searches for MSSM Higgs bosons as functions of $M_{A}$ and $\tan \beta$ in the benchmark surfaces P1, P2, P3 and P4. The Higgs bosons can either be produced 'directly' or via cascades, starting with gluino or squark production [71. We focus here on the first possibility, but it should be kept in mind that the production via cascades could offer additional channels for the Higgs detection. A full evaluation of these channels across the benchmark surfaces must await a more complete evaluation of the experimental sensitivities to such decay modes.

We start the analysis with the light MSSM Higgs boson that behaves like the SM Higgs boson for $M_{A} \gg M_{Z}$. As a consequence, the region $M_{A} \gg M_{Z}$ can be covered in all benchmark scenarios if a SM Higgs with $M_{H}^{\mathrm{SM}}=M_{h}$ is accessible at the LHC [11, 12, 14]. In figure 4 we display on the WMAP-compatible $\left(M_{A}, \tan \beta\right)$ planes the 5 - $\sigma$ discovery contours for $p p \rightarrow h \rightarrow \gamma \gamma$ at the LHC with $30 \mathrm{fb}^{-1}$ in the CMS detector [14, where the areas to the right of the lines (i.e. for larger $M_{A}$ ) are covered by the $p p \rightarrow h \rightarrow \gamma \gamma$ search. This channel is particlarly important for a precise mass measurement of the lightest MSSM Higgs boson. We show separately the sensitivities for a cut-based analysis (blue solid line) and for an "optimized" analysis (black dotted line), see ref. [14] for details. The cut-based analysis should be regarded as a conservative result, while the "optimized" analysis should perhaps be regarded as an optimistic expectation $[72$. In the cases of surfaces $\mathbf{P} \mathbf{1}$ and P2, the LHC cut analysis for the $p p \rightarrow h \rightarrow \gamma \gamma$ search covers all of the $\Delta \chi^{2}<2.30$ region

\footnotetext{
${ }^{5}$ We note that both CDF and D0 have already recorded more than $2.5 \mathrm{fb}^{-1}$ of integrated luminosity.
} 

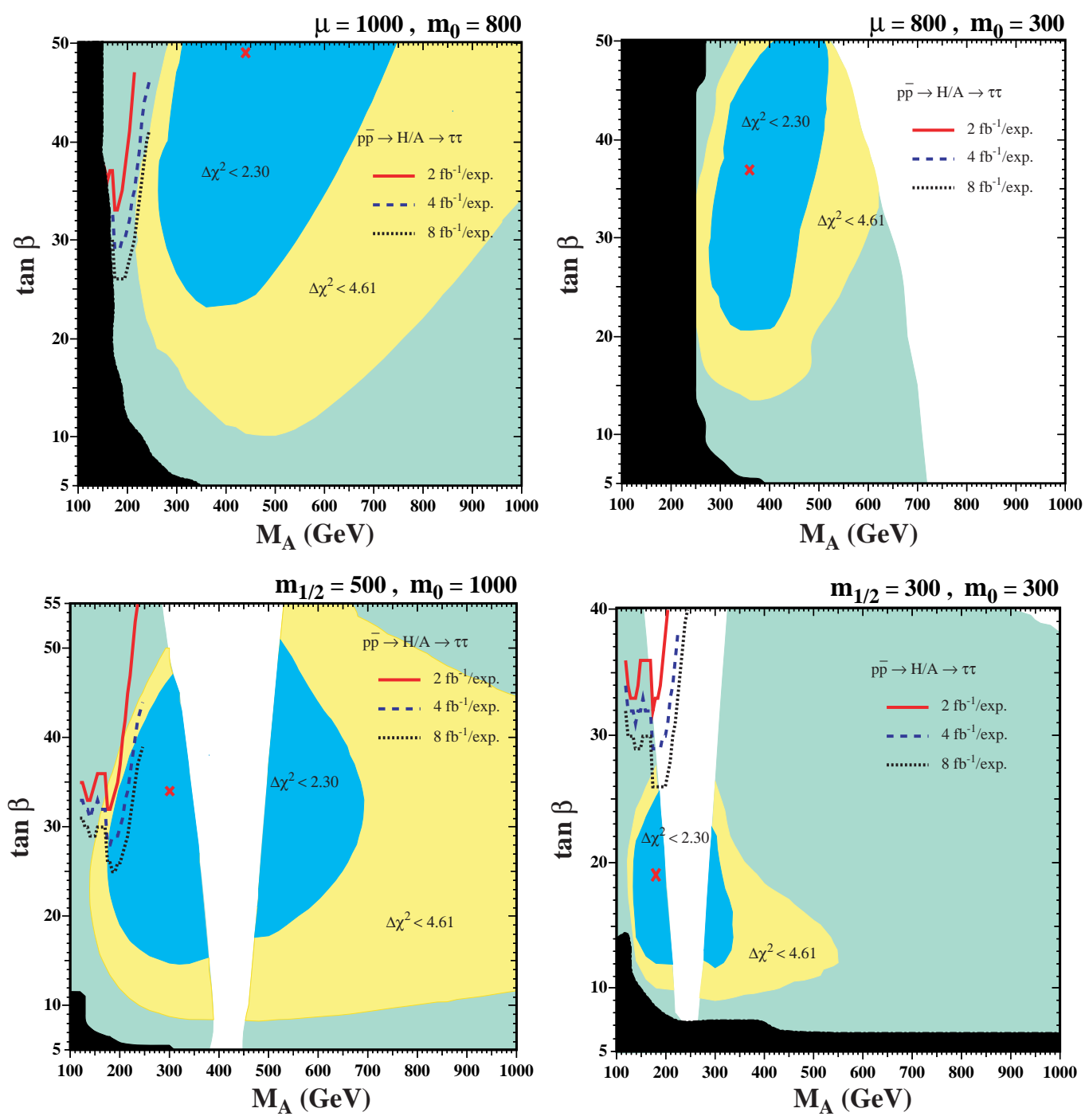

Figure 3: The same $\left(M_{A}, \tan \beta\right)$ planes for the NUHM benchmark surfaces (a) P1, (b) P2, (c) P3 and (d) P4 as in figure 2, displaying also the expected 95\% C.L. exclusion sensitivities of searches for $H / A \rightarrow \tau^{+} \tau^{-}$at the Tevatron collider with $2,4,8 \mathrm{fb}^{-1}$ in each of the CDF and D0 experiments (see text).

and the optimized analysis nearly the whole parameter plane. For P3 only parts of the preferred region can be covered, while for $\mathbf{P} \mathbf{4}$ even with the optimized analysis the best-fit point as well as large parts of the $\Delta \chi^{2}<2.30$ area remain uncovered. In this region, more luminosity would need to be accumulated in order to see a 5 - $\sigma$ signal in the $p p \rightarrow h \rightarrow \gamma \gamma$ channel.

We turn next to the reaction $W^{+} W^{-} \rightarrow h \rightarrow \tau^{+} \tau^{-}$. On the WMAP-compatible $\left(M_{A}, \tan \beta\right)$ planes in figure 1 we display the 5- $\sigma$ discovery contours for $W^{+} W^{-} \rightarrow h \rightarrow$ $\tau^{+} \tau^{-}$at the LHC with $60 \mathrm{fb}^{-1}$ in the CMS detector [14], where the areas to the right of the lines (i.e. for larger $M_{A}$ ) are covered by this search. In the cases of surfaces $\mathbf{P 1}$ and $\mathbf{P 2}$, the 

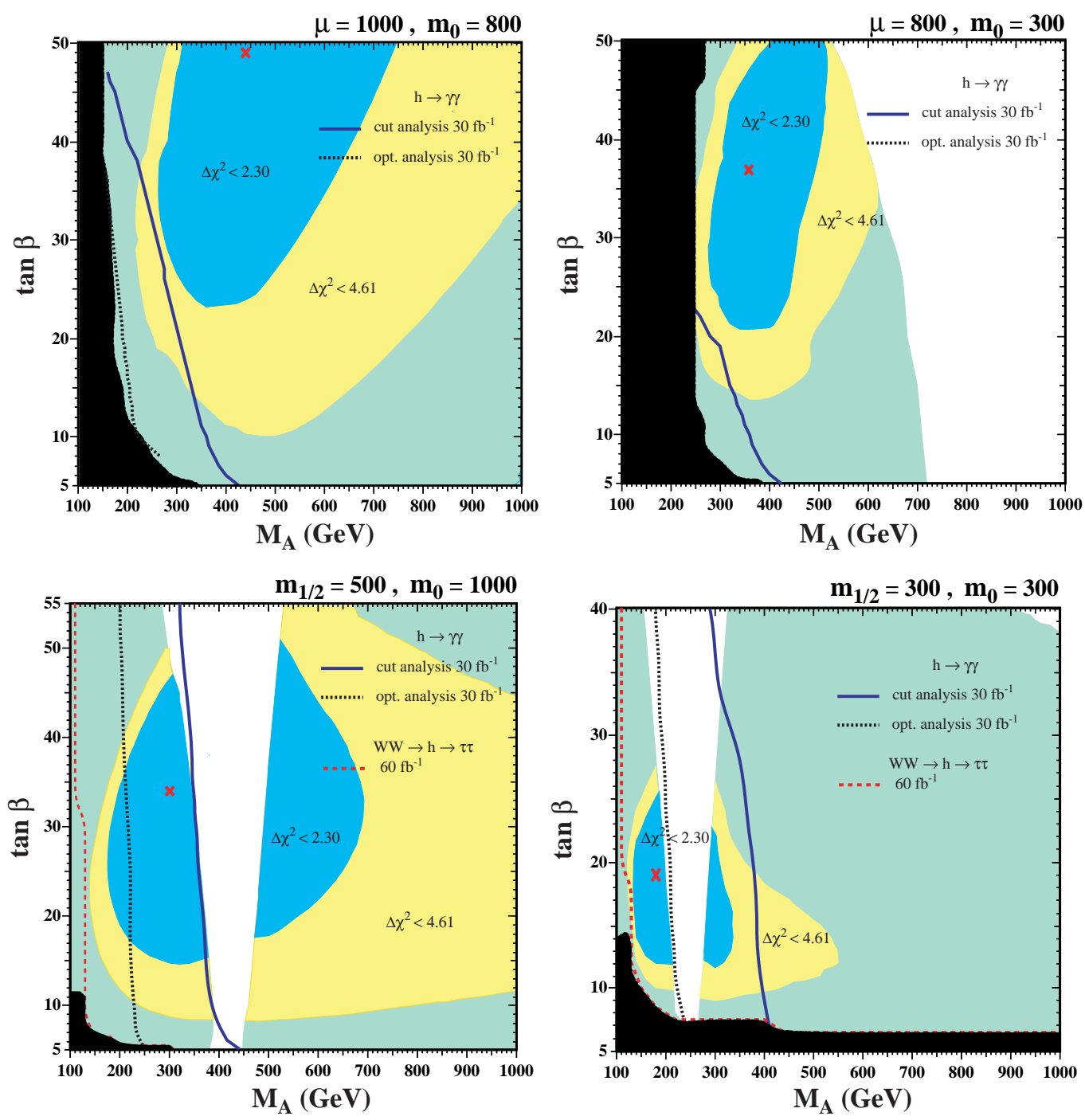

Figure 4: The same $\left(M_{A}, \tan \beta\right)$ planes for the NUHM benchmark surfaces (a) P1, (b) P2, (c) P3 and (d) P4 as in figure 2, displaying the expected sensitivities of searches for $p p \rightarrow h \rightarrow \gamma \gamma$ at the LHC with $30 \mathrm{fb}^{-1}$ in the CMS detector using a cut analysis or an "optimized" analysis (see text) as well as the searches for $W^{+} W^{-} \rightarrow h \rightarrow \tau^{+} \tau^{-}$with $60 \mathrm{fb}^{-1}$ in the CMS detector. The parameter regions to the right of the contours are covered at the $5-\sigma$ level. For P1 and P2 the $W^{+} W^{-} \rightarrow h \rightarrow \tau^{+} \tau^{-}$channel covers the whole region of the $\left(M_{A}, \tan \beta\right)$ plane that is unexcluded by LEP.

5- $\sigma$ discovery contours lie within the region already excluded by LEP, so this search covers all the unexcluded parts of the surfaces. In the cases of surfaces P3 and P4, however, the $W^{+} W^{-} \rightarrow h \rightarrow \tau^{+} \tau^{-}$discovery contours leave uncovered narrow strips at low $M_{A}$ for $\tan \beta>11,14$, respectively. In this part of the parameter space the search for $H \rightarrow \tau^{+} \tau^{-}$ should be investigated. In all cases, the $5-\sigma$ discovery contours cover the entire $\Delta \chi^{2}<4.61$ regions. However, we note that this channel does not permit a very accurate measurement 

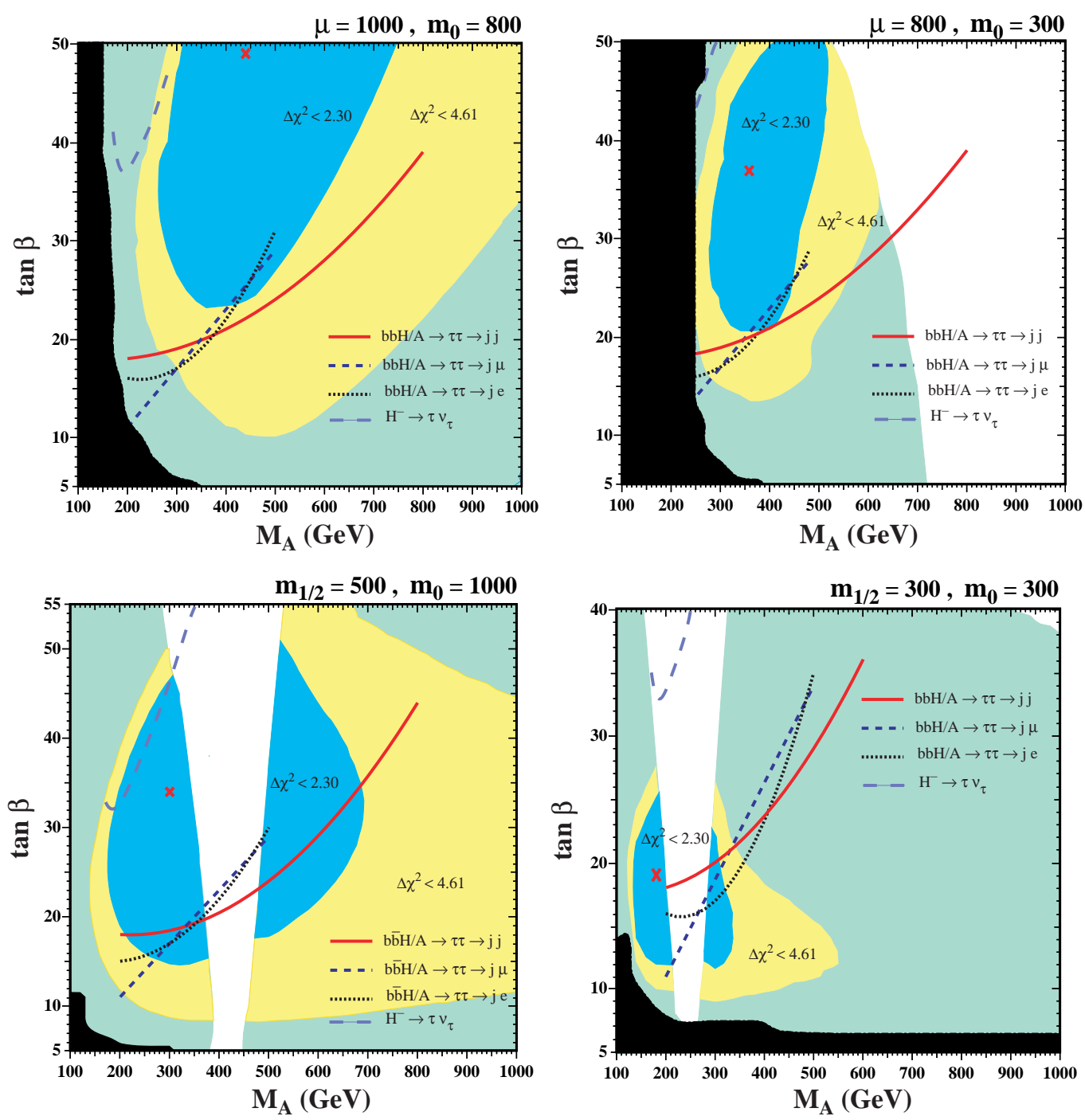

Figure 5: The same $\left(M_{A}, \tan \beta\right)$ planes for the NUHM benchmark surfaces (a) P1, (b) P2, (c) $\mathbf{P 3}$ and (d) $\mathbf{P} 4$ as in figure 2, displaying the 5- $\sigma$ discovery contours for $H / A \rightarrow \tau^{+} \tau^{-}$at the LHC with 60 or $30 \mathrm{fb}^{-1}$ (depending on the $\tau$ decay channels) and for $H^{ \pm} \rightarrow \tau^{ \pm} \nu$ detection in the CMS detector when $M_{H^{ \pm}}>m_{t}$ (see text).

of $M_{h}$, unlike the $p p \rightarrow h \rightarrow \gamma \gamma$ channel.

We now turn to the heavy MSSM Higgs bosons. In figure a we display in the $\left(M_{A}, \tan \beta\right)$ planes the $5-\sigma$ discovery contours for $b \bar{b} \rightarrow H / A \rightarrow \tau^{+} \tau^{-}$at the LHC, where the $\tau$ 's decay to jets and electrons or muons (in the BR evaluation for the heavy Higgs bosons possible decays to SUSY particles [73-75] have also been taken into account). The analysis is based on $60 \mathrm{fb}^{-1}$ for the final state $\tau^{+} \tau^{-} \rightarrow$ jets $[76]$ and on $30 \mathrm{fb}^{-1}$ for the $\tau^{+} \tau^{-} \rightarrow e+$ jet [77] and $\tau^{+} \tau^{-} \rightarrow \mu+$ jet [78] channels, collected with the CMS detector. As shown in ref. [79], the impact of the supersymmetric parameters other than $M_{A}$ and $\tan \beta$ on the discovery contours is relatively small in this channel, and the decays of $H / A$ 
to SUSY particles [73- 75] are in general suppressed by large sparticle masses. Only in $\mathbf{P 4}$ the decay to the lightest neutralinos and charginos is possible over nearly the whole plane (see also section 2). Including such decays in the evaluation of the discovery reach could increase the coverage for heavy Higgs bosons somewhat. As a consequence of the relatively small impact of the other SUSY parameters, the discovery contours in the four benchmark surfaces are similar to each other and to those in the "conventional" benchmark scenarios [79]. The 5- $\sigma$ discovery contours for the various $\tau$ decay modes are shown separately: they may each be scaled individually for different values of the jet $(\mathrm{j}), \mu$ and electron $(e)$ detection efficiencies, see ref. [79]. The sensitivities of the three different search strategies could in principle be combined, but information required for making such a combination is not yet available from the CMS Collaboration. Nor is the information available that would be needed to extend the discovery contours to small $M_{A}<200 \mathrm{GeV}$ or to large $M_{A}>500$ to $800 \mathrm{GeV}$. Nevertheless, we see that the whole $\Delta \chi^{2}<2.30$ regions of the surfaces $\mathbf{P} 1$ and P2 would be covered by the LHC $H / A \rightarrow \tau^{+} \tau^{-}$searches, and most of the corresponding regions of the surfaces $\mathbf{P 3}$ and $\mathbf{P 4}$. Comparing the LHC sensitivities shown in figure 0 with the Tevatron sensitivities shown in figure 3, we see that the LHC provides access to considerably heavier $H / A$, up to about $800 \mathrm{GeV}$, and that the covered region extends to

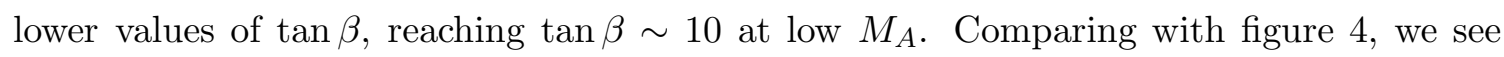
that the $H / A \rightarrow \tau^{+} \tau^{-}$searches presumably also cover the regions at $M_{A}<150 \mathrm{GeV}$ and $\tan \beta>11,14$ that were left uncovered in the $\mathbf{P 3}$ and $\mathbf{P} 4$ surfaces, respectively, by the $W^{+} W^{-} \rightarrow h \rightarrow \tau^{+} \tau^{-}$searches. It would be interesting to verify this by means of an extension of the available CMS analysis.

We also show in figure 5 the 5 - $\sigma$ contours for discovery of the $H^{ \pm}$via its $\tau^{ \pm} \nu$ decay mode at the LHC, in the case $M_{H^{ \pm}}>m_{t}$. We see that the coverage is limited in each of the scenarios P1, P2, P3 and $\mathbf{P} 4$ to $M_{A}<300 \mathrm{GeV}$ and $\tan \beta>30$, reaching a small part of the $\Delta \chi^{2}<2.30$ region of surface $\mathbf{P 3}$, only a small part of the $\Delta \chi^{2}<4.61$ region of surface $\mathbf{P 1}$, and not even reaching this region in scenarios $\mathbf{P 2}$ and $\mathbf{P 4}$. One may also search for $H^{ \pm} \rightarrow \tau^{ \pm} \nu$ for lighter $M_{H^{ \pm}}<m_{t}$, but in the cases of surfaces $\mathbf{P} 1$ and $\mathbf{P} 2$ this would be useful only in the regions already excluded by LEP, and the accessible regions in surfaces $\mathbf{P 3}$ and $\mathbf{P} 4$ would also be quite limited.

Another class of possible measurements at the LHC comprises the precise determinations of $h$ decay branching ratios 80, and using their ratios to search for deviations from the SM predictions for a Higgs boson of the same mass. Such deviations may arise in the MSSM due to differences in the tree-level couplings and due to additional (loop) corrections. The most sensitive observable is likely to be the ratio of $\operatorname{BR}\left(h \rightarrow \tau^{+} \tau^{-}\right) / \operatorname{BR}\left(h \rightarrow W W^{*}\right)$. We display in figure 6 the 1-, 2-, 3- and 5- $\sigma$ contours (2- $\sigma$ in bold) for SUSY induced deviations of this ratio of branching ratios from the SM prediction (with $M_{H}^{\mathrm{SM}}=M_{h}$ ). The contours correspond to an integrated luminosity at the LHC of 30 or $300 \mathrm{fb}^{-1}$ [81] (assuming SM decay rates). An experimental resolution for $\operatorname{BR}\left(h \rightarrow \tau^{+} \tau^{-}\right) / \operatorname{BR}\left(h \rightarrow W W^{*}\right)$ between $30 \%(28 \%)$ and $45 \%(33 \%)$ can be achieved for $30(300) \mathrm{fb}^{-1}$. For $M_{h}=120 \mathrm{GeV}$ the corresponding precision is $38 \%(29 \%)$. The most promising surfaces are P3 and P4, and we see that over essentially all the left lobe of the $\Delta \chi^{2}<4.61$ region for $\mathbf{P} 4$ a $5-\sigma$ 

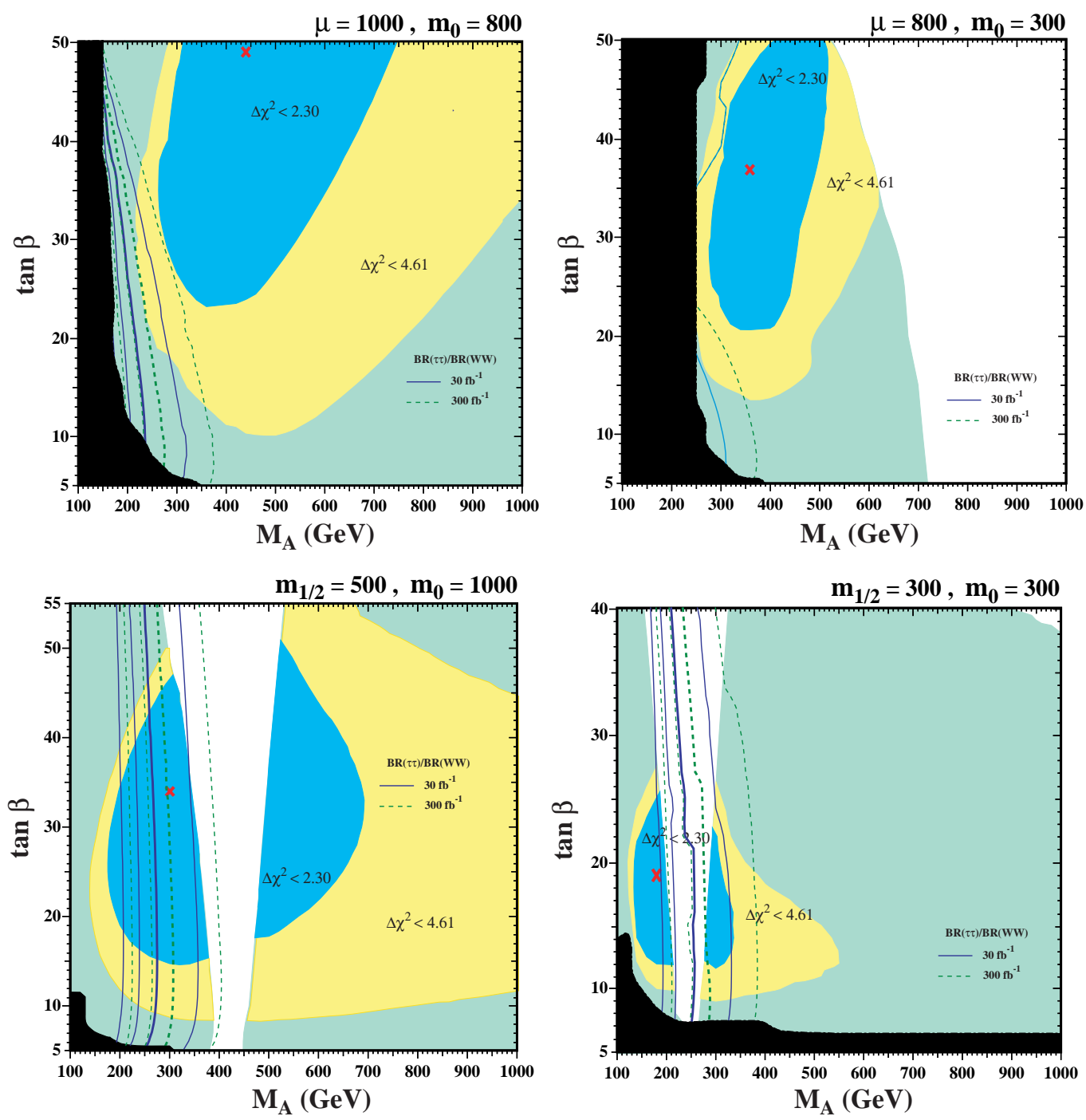

Figure 6: The same $\left(M_{A}, \tan \beta\right)$ planes for the NUHM benchmark surfaces (a) P1, (b) P2, (c) P3 and (d) $\mathbf{P} 4$ as in figure 2, displaying the 1-, 2-, 3- and 5- $\sigma$ contours (2- $\sigma$ in bold) for SUSY-induced deviations on the ratio $\mathrm{BR}\left(h \rightarrow \tau^{+} \tau^{-}\right) / \mathrm{BR}\left(h \rightarrow W W^{*}\right)$ at the LHC with 30 or $300 \mathrm{fb}^{-1}$ (see text). In the case of surface $\mathbf{P 2}$, only 1- $\sigma$ curves are seen in the lower part of the figure. The upper curves correspond to $0-\sigma$.

discrepancy with the SM should be detectable. ${ }^{6}$ On the other hand, only partial coverage of the left lobe of surface P3 would be possible, and the sensitivities in the right lobes of $\mathbf{P} \mathbf{4}$ and $\mathbf{P 3}$ and in the $\mathbf{P 1}$ and P2 surfaces are considerably less promising. Nevertheless, measuring $\operatorname{BR}\left(h \rightarrow \tau^{+} \tau^{-}\right) / \mathrm{BR}\left(h \rightarrow W W^{*}\right)$ does offer the prospect of distinguishing between the NUHM and the SM in the low $M_{A}$ regions of surfaces $\mathbf{P 3}$ and $\mathbf{P} 4$.

\footnotetext{
${ }^{6}$ It should be kept in mind that the actual experimental precision on the ratio $\operatorname{BR}\left(h \rightarrow \tau^{+} \tau^{-}\right) / \operatorname{BR}(h \rightarrow$ $\left.W W^{*}\right)$ will be different in this parameter region from the numbers quoted above which assume SM rates.
} 


\begin{tabular}{|c|c|c|}
\hline collider & channel & exp. precision [\%] \\
\hline $\mathrm{ILC}(500)$ & $\mathrm{BR}(h \rightarrow b \bar{b})$ & 1.5 \\
\hline $\mathrm{ILC}(500)$ & $\mathrm{BR}\left(h \rightarrow \tau^{+} \tau^{-}\right)$ & 4.5 \\
\hline $\mathrm{ILC}(500)$ & $\mathrm{BR}\left(h \rightarrow W W^{*}\right)$ & 3.0 \\
\hline $\mathrm{ILC}(1000)$ & $\mathrm{BR}(h \rightarrow b \bar{b}) / \mathrm{BR}\left(h \rightarrow W W^{*}\right)$ & 1.5 \\
\hline
\end{tabular}

Table 2: Experimental precisions at the ILC for various branching ratios of the lightest MSSM Higgs boson (assuming SM decay rates) 82, 18, 83. The experimental precision in the last column corresponds to $1 \sigma$ in the plots below. ILC $(500,1000)$ refers to a center-of-mass energy of $500,1000 \mathrm{GeV}$, respectively.

\section{ILC phenomenology}

In this section we analyze the deviations in the branching ratios of the lightest MSSM Higgs boson to SM fermions and gauge bosons in comparison with a SM Higgs boson of the same mass that could be measured at the ILC (see also ref. [21]). The experimental precisions for the branching ratios we analyze are summarized in table 2 .

We show in figure 7 the prospective sensitivity of an ILC measurement of the $\mathrm{BR}(h \rightarrow$ $b \bar{b})$ in the four $\left(M_{A}, \tan \beta\right)$ planes. The experimental precision is anticipated to be $1.5 \%$, see table 2. We display as solid (blue) lines the contours of the $+5,+3,+2,+1,0 \sigma$ deviations (with $+2 \sigma$ in bold) of the MSSM result from the corresponding SM result (for low $M_{A}$ and large $\tan \beta$ in $\mathbf{P 2}$ we also find contours for $-2,-1 \sigma$, with $-2 \sigma$ in bold). The separations between the contours indicate how sensitively the SUSY results depend on variations of $M_{A}$ and $\tan \beta$. Also shown in figure 7 via dashed (green) lines is the sensitivity to SUSY effects of the ILC measurement of the ratio of branching ratios $\mathrm{BR}(h \rightarrow b \bar{b}) / \mathrm{BR}\left(h \rightarrow W W^{*}\right)$ (for low $M_{A}$ and large $\tan \beta$ in $\mathbf{P 2}$ we also find contours for $\left.-5,-3,-2,-1 \sigma\right)$. The precision measurement of the ratio $\mathrm{BR}(h \rightarrow b \bar{b}) / \mathrm{BR}\left(h \rightarrow W W^{*}\right)$ clearly provides a much higher sensitivity to SUSY effects than the measurement of $\operatorname{BR}(h \rightarrow b \bar{b})$ alone (see also ref. [20]).

For the ILC measurement of the $\mathrm{BR}(h \rightarrow b \bar{b})$, in the cases of $\mathbf{P 1}$ and $\mathbf{P 2}$ we see that the prospective sensitivities are less than $3 \sigma$ throughout almost all the regions with $\Delta \chi^{2}<4.61$. The situations are different, however, for the planes $\mathbf{P 3}$ and $\mathbf{P 4}$. In each case, the cosmologically-favoured region is divided into separate lobes at low and high $M_{A}$. In the P3 case, the measurement of $\mathrm{BR}(h \rightarrow b \bar{b})$ would be sufficient to establish a SUSY effect with more than five $\sigma$ throughout most of the low- $M_{A}$ lobe, and all of it in the $\mathbf{P} 4$ case. A precision measurement of $\mathrm{BR}(h \rightarrow b \bar{b}) / \mathrm{BR}\left(h \rightarrow W W^{*}\right)$ yields a significant improvement for all benchmark surfaces. We see that, in case $\mathbf{P 1}$, the sensitivity already exceeds $5 \sigma$ in much of the region with $\Delta \chi^{2}<2.30$, and the fraction of this region covered at the 5- $\sigma$ level is even larger in the case $\mathbf{P 2}$. Even more encouragingly, in the case $\mathbf{P} \mathbf{3}$ the sensitivity exceeds $5 \sigma$ throughout the $\Delta \chi^{2}<2.30$ region, and in the case $\mathbf{P} 4$ it exceeds $5 \sigma$ by a substantial amount throughout the $\Delta \chi^{2}<4.61$ region.

Next, we show in figure 8 the prospective sensitivity of an ILC measurement of the $\operatorname{BR}\left(h \rightarrow \tau^{+} \tau^{-}\right)$in the four $\left(M_{A}, \tan \beta\right)$ planes, using solid (red) contours. In the cases of $\mathbf{P 1}$ and $\mathbf{P 2}$, we again see that the prospective sensitivities are less than $3 \sigma$ throughout almost 

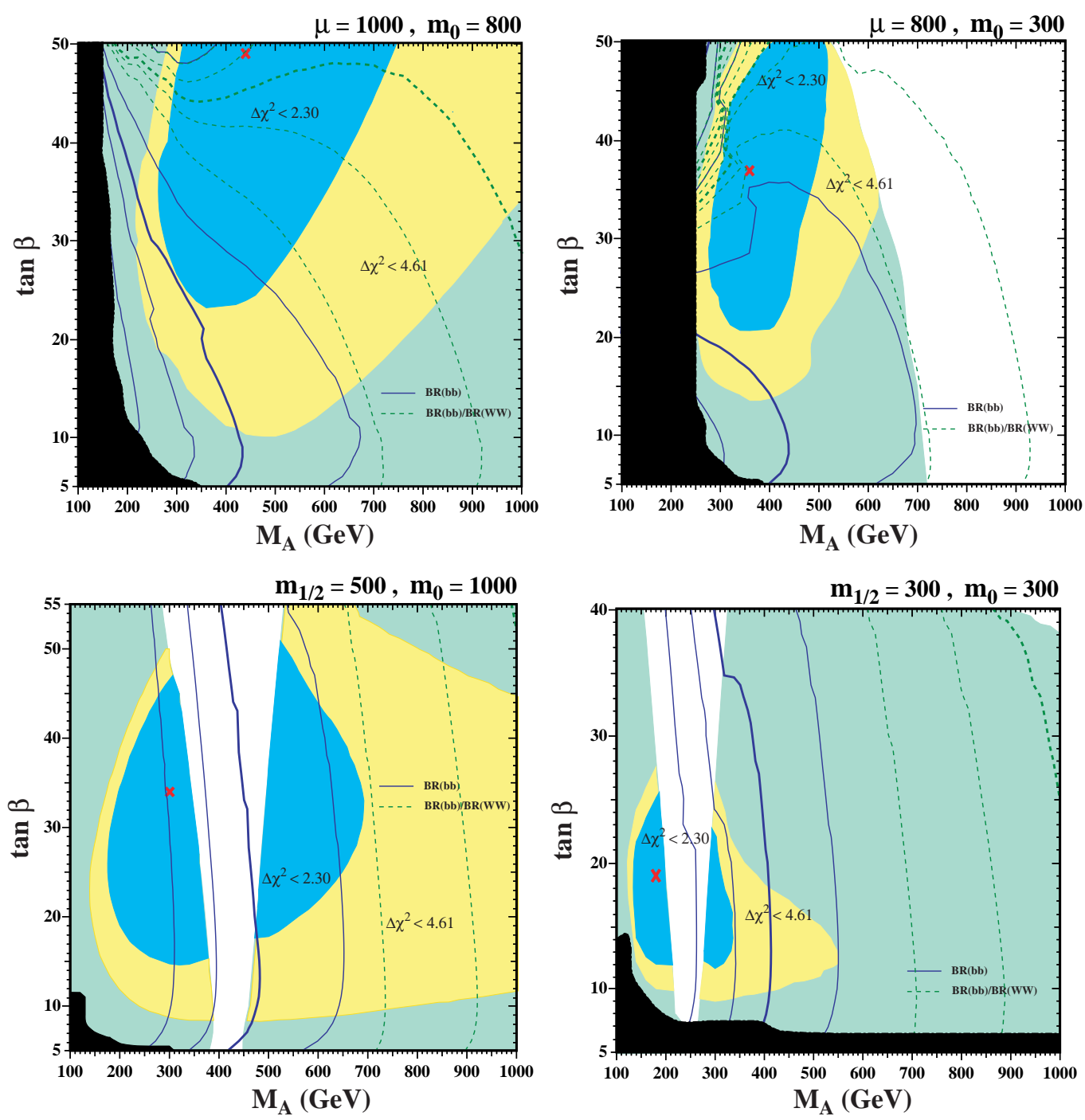

Figure 7: The same $\left(M_{A}, \tan \beta\right)$ planes for the NUHM benchmark surfaces (a) P1, (b) P2, (c) P3 and (d) P4 as in figure 2, displaying 5,3,2,1,0- $\sigma$ sensitivity contours (2- $\sigma$ in bold) for SUSY effects on $\mathrm{BR}(h \rightarrow b \bar{b})$ (solid blue lines) and $\mathrm{BR}(h \rightarrow b \bar{b}) / \mathrm{BR}\left(h \rightarrow W W^{*}\right)$ (dashed green lines) at the ILC (see text). Note that for surface P2 for low $M_{A}$ and large $\tan \beta$ also $-2,-1-\sigma$ are shown for $\operatorname{BR}(h \rightarrow \bar{b} b)$, and $-5,-3,-2,-1-\sigma$ are shown for $\operatorname{BR}(h \rightarrow \bar{b} b) / \mathrm{BR}\left(h \rightarrow W W^{*}\right)$.

all the regions with $\Delta \chi^{2}<4.61$. In the cases of planes $\mathbf{P 3}$ and $\mathbf{P 4}$, the sensitivities are greater, but less than the corresponding sensitivities to the $\mathrm{BR}(h \rightarrow b \bar{b})$ shown previously in figure 0. Of all the single ILC measurements, the one with the greatest sensitivity to SUSY effects is that of the $\mathrm{BR}\left(h \rightarrow W W^{*}\right)$, which is also shown in figure 8 using dashed (black) lines. In the cases $\mathbf{P} \mathbf{1}$ and $\mathbf{P 2}$, we see that the sensitivity may rise above $5 \sigma$ already within the $\Delta \chi^{2}<4.61$ region. In the case of $\mathbf{P 3}$, the sensitivity is well above $5 \sigma$ throughout the low- $M_{A}$ region. In the case of $\mathbf{P 4}$, a $5-\sigma$ significance is exceeded already in much of the high- $M_{A}$ lobe, where the sensitivity never falls as low as $3 \sigma$ in the $\chi^{2}$ favored 

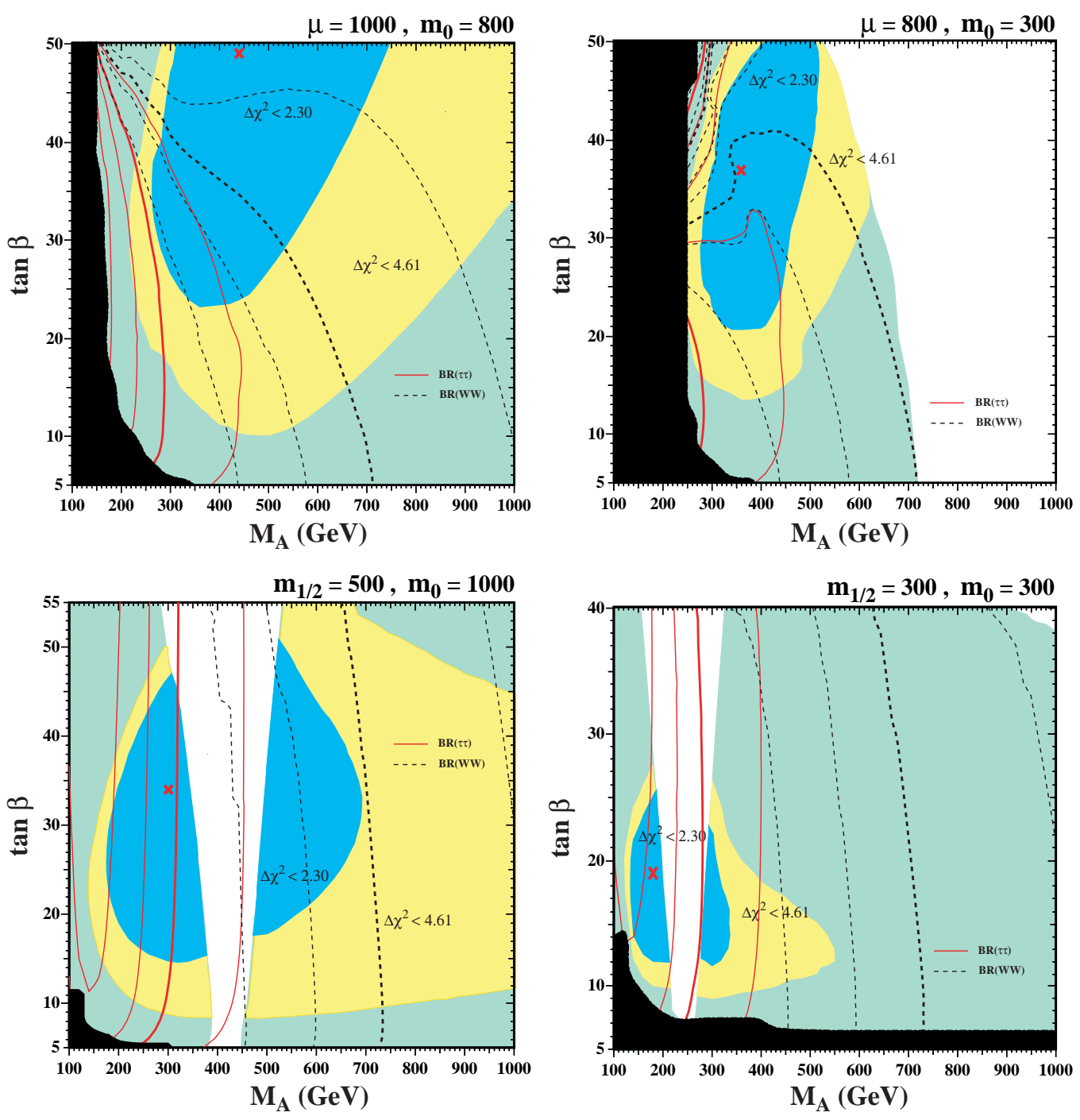

Figure 8: The same $\left(M_{A}, \tan \beta\right)$ planes for the NUHM benchmark surfaces (a) P1, (b) P2, (c) P3 and (d) P4 as in figure 2, displaying 5,3,2,1- $\sigma$ sensitivity contours for SUSY effects on the $\operatorname{BR}\left(h \rightarrow \tau^{+} \tau^{-}\right)$at the ILC (solid red lines). Also shown are the $-5,-3,-2,-1-\sigma$ sensitivity contours for the SUSY effects on $\operatorname{BR}\left(h \rightarrow W W^{*}\right)$ at the ILC (dashed black lines). Note that for surface $\mathbf{P 2} \pm 2, \pm 1$ and $0-\sigma$ are shown for $\operatorname{BR}\left(h \rightarrow \tau^{+} \tau^{-}\right)$, and $\pm 5, \pm 3, \pm 2, \pm 1$ and 0 - $\sigma$ are shown for $\operatorname{BR}\left(h \rightarrow W W^{*}\right)$.

region.

We have not made a complete study of the combined sensitivity of the ILC measurements to the benchmark surfaces, but it is clear from this brief survey that the ILC measurements would in general provide interesting tests of the MSSM at the loop level. In the absence of detailed studies, we expect that CLIC measurements would have similar sensitivities, since $h$ production would be more copious at the higher CLIC energies, and the CLIC luminosity at lower energies could be similar to that of the ILC [23]. In addition 
to the precision measurements described here, the ILC and CLIC would be able to produce directly associated $H+A$ pairs above the kinematic threshold.

\section{7. $B$ physics}

We display in figure 9 the results for three $\mathrm{BPO} \mathrm{BR}(b \rightarrow s \gamma), \mathrm{BR}\left(B_{s} \rightarrow \mu^{+} \mu^{-}\right), \mathrm{BR}\left(B_{u} \rightarrow\right.$ $\left.\tau \nu_{\tau}\right)$, in the four benchmark $\left(M_{A}, \tan \beta\right)$ planes.

The prediction of $B_{s} \rightarrow \mu^{+} \mu^{-}$is based on ref. [84, 47]. The solid (beige) line indicates $\mathrm{BR}\left(B_{s} \rightarrow \mu^{+} \mu^{-}\right)=10^{-7}$, corresponding roughly to the current upper bound from CDF 855 and D0 [86]. The latest bound reported by $\mathrm{CDF}$ has recently been lowered to $5.8 \times 10^{-8} 87$. The dashed (beige) line indicates a BR of $2 \times 10^{-8}$. In figure 9 we see that the current upper limit on $B_{s} \rightarrow \mu^{+} \mu^{-}$already excludes regions of the planes at small $M_{A}$ and large $\tan \beta$, starting to cut into the region with $\Delta \chi^{2}<4.61$. The prospective sensitivities would extend as far as the best-fit points.

For $b \rightarrow s \gamma$ our numerical results have been derived with the $\mathrm{BR}(b \rightarrow s \gamma)$ evaluation provided in refs. [88], incorporating also the latest SM corrections provided in ref. [89]. The results in figure 9 are shown as the two blue lines indicating $\operatorname{BR}(b \rightarrow s \gamma)$ of $4 \times 10^{-4}$ (solid) and $3 \times 10^{-4}$ (dashed). These have to be compared to the experimentally preferred value of $\operatorname{BR}(b \rightarrow s \gamma)=\left(3.55 \pm 0.24_{-0.10}^{+0.09} \pm 0.03\right) \times 10^{-4}$ [90]. The best-fit point together with large parts of the $\chi^{2}$ preferred regions lie between the two lines, i.e., large parts of the four benchmark planes are in good agreement with the current experimental value.

Our results for $\mathrm{BR}\left(B_{u} \rightarrow \tau \nu_{\tau}\right)$ are based on ref. [91]. In the four benchmark scenarios of figure 9 the results are shown in form of the NUHM result divided by the SM prediction as black lines. The solid (dashed) lines correspond to a ratio of $0.9(0.7)$, where the current central value is $0.93 \pm 0.41$ [92, 93]. It can be seen that the best fit value as well as large parts of the $\chi^{2}$ preferred parts of the benchmark planes predict a value somewhat lower than the current experimental result. However, with the current precision no firm conclusion can be drawn.

\section{Direct detection of supersymmetric dark matter}

In figure 10 we show how the direct detection of the LSP via spin-independent scattering on nuclei probes the four $\left(M_{A}, \tan \beta\right)$ planes. We focus here on the bound from the XENON10 experiment that was recently published by the XENON collaboration [27], which improves on the previous CDMS results [26]. We note that the XENON10 experiment has seen some potential signal events which are, however, interpreted as background.

The constraint imposed by the limits from direct detection experiments is sensitive to two theoretical uncertainties that are independent of the specific dark matter model. One is the local density of cold dark matter, which is normally estimated to be $\rho_{\mathrm{CDM}}=0.3 \mathrm{GeV} / \mathrm{cm}^{3}$, although smaller values may be consistent with some models of the Galaxy. The other important uncertainty is that in the nucleonic matrix element of the local operator responsible for the spin-independent scattering amplitude. This is related, 

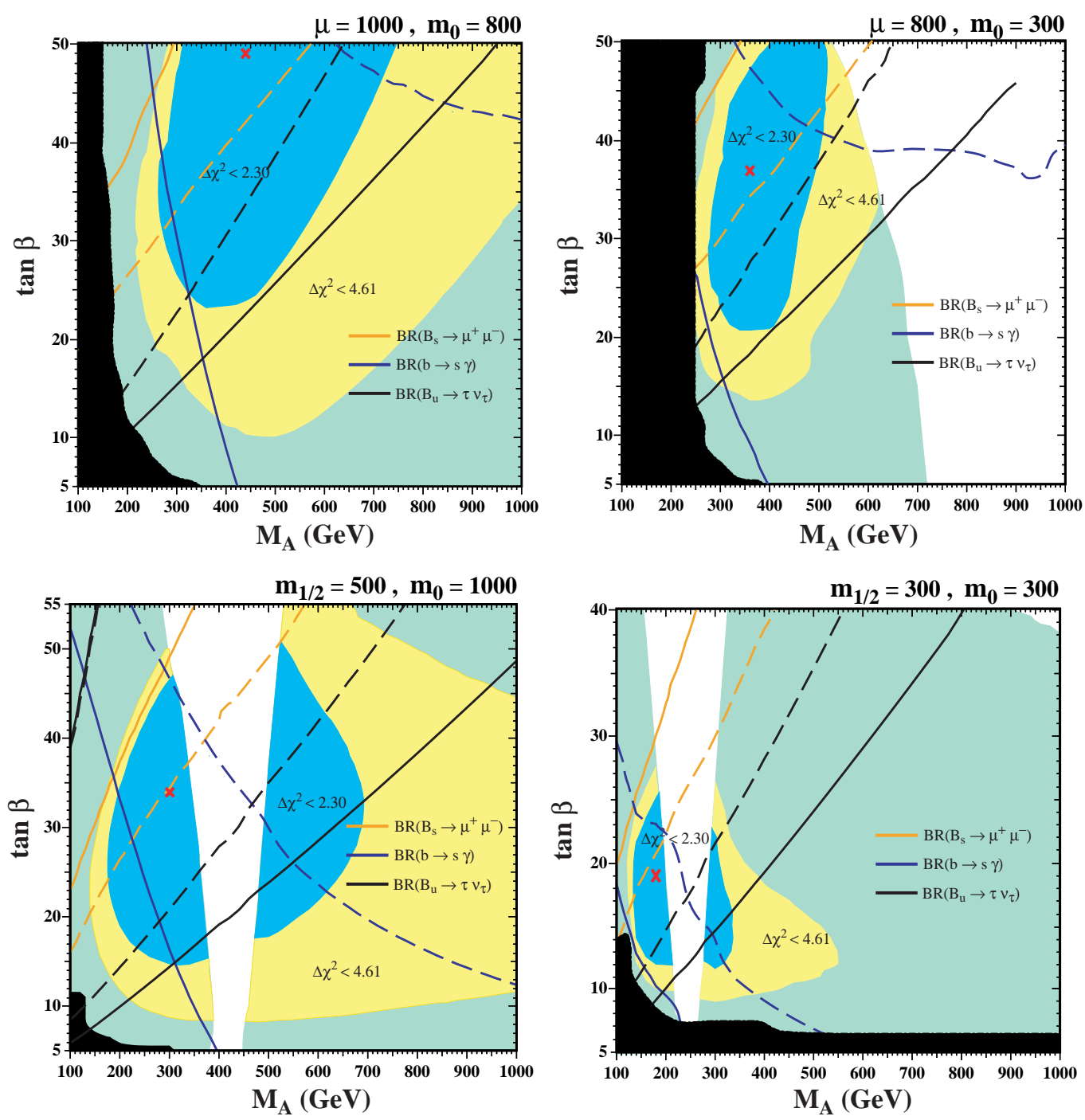

Figure 9: The same $\left(M_{A}, \tan \beta\right)$ planes for the NUHM benchmark surfaces (a) P1, (b) P2, (c) P3 and (d) P4 as in figure 2, displaying the expected sensitivities of the $B$ physics observables $B_{s} \rightarrow \mu^{+} \mu^{-}, b \rightarrow s \gamma$ and $B_{u} \rightarrow \tau \nu$. The various lines indicate: $\operatorname{BR}\left(B_{s} \rightarrow \mu^{+} \mu^{-}\right)=10^{-7}\left(2 \times 10^{-8}\right)$ as solid (dashed), $\mathrm{BR}(b \rightarrow s \gamma)=4(3) \times 10^{-4}$ as solid (dashed), $\mathrm{BR}\left(B_{u} \rightarrow \tau \nu_{\tau}\right)_{\mathrm{MSSM} / \mathrm{SM}}=0.9(0.7)$ as solid (dashed).

in particular, to the so-called $\sigma$ term, $\Sigma_{\pi N}$, that may be derived from measurements of low-energy $\pi$-nucleon scattering.

The solid lines in figure 10 correspond to the XENON10 bound obtained assuming $\rho_{\mathrm{CDM}}=0.3 \mathrm{GeV} / \mathrm{cm}^{3}$ and using $\Sigma_{\pi N}=45 \mathrm{MeV}$ as input, corresponding to a relative strange-quark density $y \equiv 2\langle N|\bar{s} s| N\rangle /\langle N|(\bar{u} u+\bar{d} d)| N\rangle=0.2$ [94]. These assumptions are realistic, though there is a large uncertainty in the strangeness contribution which may lead to larger rates if $\Sigma_{\pi N}$ is larger or significantly lower rates if the strangeness contribution to the proton mass is small. The dashed lines show the bounds that one would obtain from 

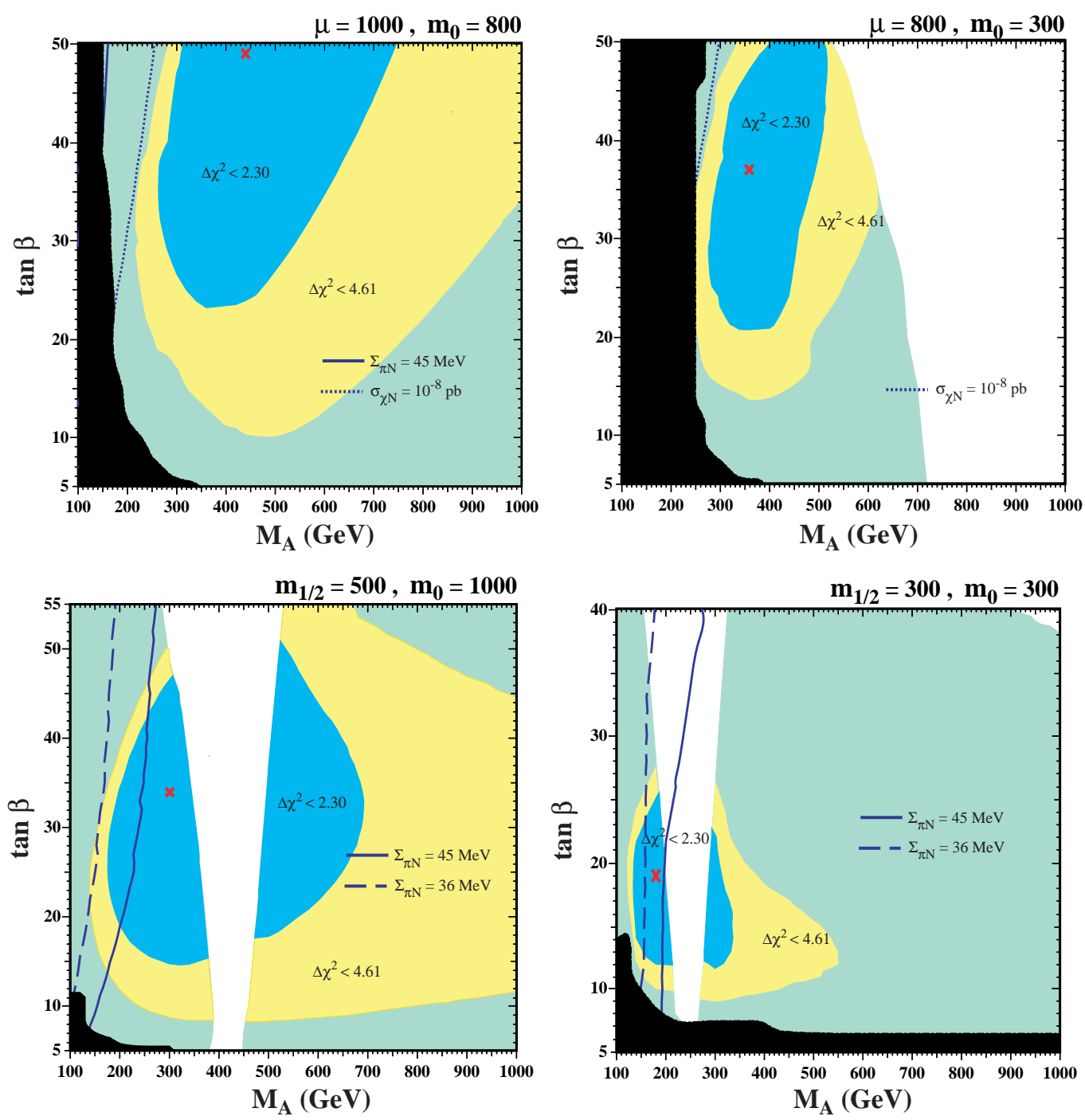

Figure 10: The same $\left(M_{A}, \tan \beta\right)$ planes for the NUHM benchmark surfaces (a) P1, (b) P2, (c) P3 and (d) P4 as in figure 2, displaying the expected sensitivities of present and prospective future direct searches for the scattering of dark matter particles (see text).

the XENON10 experiment assuming the same value of $\rho_{\mathrm{CDM}}$, but with $\Sigma_{\pi N}=36 \mathrm{MeV}$ corresponding to $y=0$, and therefore representing more conservative assumptions. Finally, as an example of the possible sensitivity of future experiments, the dotted lines show the contours one would obtain for a spin-independent cross section of $10^{-8} \mathrm{pb}$, assuming the same value of $\rho_{\mathrm{CDM}}$ and $\Sigma_{\pi N}=45 \mathrm{MeV}$ as input.

We see from figure 10 that the surfaces $\mathbf{P} \mathbf{1}$ and $\mathbf{P 2}$ are not probed by the current limits from the XENON10 experiment. Only the possible future sensitivity at $10^{-8} \mathrm{pb}$ starts to cut into the $\Delta \chi^{2}<4.61$ region. For these planes, accelerator searches are clearly more powerful. The situation is different for the planes $\mathbf{P 3}$ and $\mathbf{P 4}$, due to the relatively low 
values of $m_{1 / 2}$ across these planes. We recall that, for planes $\mathbf{P} \mathbf{1}$ and $\mathbf{P 2}, m_{1 / 2}$ scales with $M_{A}$ and the sparticle spectrum is typically heavier at large $M_{A}$ than at the corresponding points in planes P3 and $\mathbf{P 4}$. As a result, the spin-independent $\tilde{\chi}_{1}^{0}-p$ elastic cross section is suppressed for planes $\mathbf{P 1}$ and P2. On the other hand, we see that the current XENON10 bound probes large parts of the $\Delta \chi^{2}<2.30$ areas of $\mathbf{P 3}$ and $\mathbf{P} 4$ planes, if one uses the moderate values of $\Sigma_{\pi N}=45 \mathrm{MeV}$ and the strange-quark content. Indeed, in the case of the P4 surface, the current XENON10 bound would even cover the best-fit point for this value of $\Sigma_{\pi N}$ and the default value for the local density of cold dark matter. The more conservative analysis, on the other hand, is sensitive only to smaller $M_{A}$ values, and probes only a much smaller part of the regions preferred by the $\chi^{2}$ analysis. Finally, we note that a future sensitivity to a cross section of $10^{-8}$ pb would cover the entire P3 and P4 surfaces.

\section{Conclusions}

The value of benchmark studies is that they allow one to understand better the range of possibilities opened up by supersymmetry. It is therefore desirable that benchmarks be chosen in such a way as to respect, as far as possible, the definitive experimental constraints, and also that they be susceptible to systematic study. We have demonstrated in this paper how NUHM benchmark surfaces chosen so that the relic cold dark matter density falls within or below the range favoured by WMAP and other experiments may be used to probe supersymmetric phenomenology. Our approach based on the NUHM scenario significantly differs from previous proposals of benchmark scenarios for the MSSM Higgs sector that were entirely formulated in terms of low-scale parameters and that were not suitable for a phenomenologically acceptable prediction of the cold dark matter density. The analysis of our proposed benchmark surfaces is facilitated by developments in the FeynHiggs code that are described in the appendix. These will enable the interested reader to explore the prospects for her/his favourite experimental probe of supersymmetry in these benchmark surfaces.

We have displayed the constraints currently imposed in the new benchmark surfaces by electroweak precision observables, and explored the prospects for Higgs searches at the Tevatron collider, the LHC and the ILC, and we have also explored indirect effects in $B$ physics and in dark matter detection. Whereas the Tevatron collider will be able only to nibble at corners of these NUHM benchmark surfaces, experiments at the LHC will be able to cover them entirely, and the ILC will have good prospects for precision measurements. There are good prospects for $B$ experiments in parts of the benchmark surfaces, and direct dark matter may be detectable in some cases.

It should of course be noted that benchmark studies may soon be rendered obsolete - namely by the discovery of supersymmetry.

As we were completing this paper, we heard the sad news of the passing away of Julius Wess, one of the discoverers and founding fathers of supersymmetry. Julius did so much to develop our understanding of supersymmetry, to awaken our appreciation of its beauty, and to convince us of its importance for physics. Humbly and respectfully, we dedicate this paper to his memory. 


\section{Acknowledgments}

S.H. thanks R. Kinnunen for data on the charged Higgs-boson search at CMS, A. Lath for communication on the CDF projections, A. Nikitenko for the CMS data on the Higgs decay to photons, and A. Korytov and E. Yazgan for information on the $W W$ fusion channels at CMS.

The work of K.A.O. was partially supported by DOE grant DE-FG02-94ER-40823. Work supported in part by the European Community's Marie-Curie Research Training Network under contract MRTN-CT-2006-035505 'Tools and Precision Calculations for Physics Discoveries at Colliders'

\section{A. Evaluation of benchmark surfaces with FeynHiggs}

The new benchmark surfaces have been implemented into the code FeynHiggs [55, 56, 54, 57. In this way, any user may apply them to perform phenomenological analyses.

From the mathematical point of view, the NUHM/CDM constraints introduce nontrivial relations between input parameters, which thus cannot be scanned naively by independent loops. To solve this in a generic way, FeynHiggs 2.6 allows the user to interpolate the inputs from a Parameter table into which arbitrary relations can be encoded. The tables containting the four benchmark surfaces can be downloaded from http://www.feynhiggs.de. To implement the new format of a Parameter table, significant internal rearrangements were necessary from which the concept of a FeynHiggs Record evolved.

A Record is a new data type which captures the entire content of a parameter file in the native format of FeynHiggs. In this respect it is akin to the SUSY Les Houches Accord Record [95], but also encodes information about parameter loops and has 'inheritance rules' for default values. Using the routines to manipulate a Record, the programmer can, among other things, process FeynHiggs parameter files independently of the front end.

In addition to containing loops over parameters, a Record can be associated with a Parameter table in such a way that values not explicitly given in the parameter file are interpolated from the table (as it can be done for the four benchmark scenarios).

The FeynHiggs Record is conceptually a superstructure 'on top' of the conventional part of FeynHiggs. This means that a Record can be manipulated without any influence on the computation of Higgs observables at first. Only when the FHSetRecord subroutine is invoked are its current values set as the inputs for the computation. So in principle, the FeynHiggs Record can be used without doing any computation of Higgs observables at all.

Technically, a Record is a two-dimensional real array of the form

\begin{tabular}{|l|cccc|}
\hline $\operatorname{rec}\left(i_{\downarrow}, j \rightarrow\right)$ & iVar & iLower & iUpper & iStep \\
\hline iTB & $L$ & $U$ & $U$ & $U$ \\
iMAO & $L$ & $U$ & $U$ & $U$ \\
$\ldots$ & $\ldots$ & & & \\
\hline
\end{tabular}

- The column index $i$ specifies the parameter. The indices are labelled as in the parameter file, but prefixed with an i (see table 3). 
- The row index $j$ enumerates the variables that constitute the loop over a parameter, i.e. the current, lower, and upper value and the step size. The loop inferred through these parameters has the form

do $\operatorname{rec}(i$, iVar $)=\operatorname{rec}(i$, iLower $), \operatorname{rec}(i$, iUpper $), \operatorname{rec}(i$, iStep $)$ ...

enddo

- $U$ entries indicate fields filled in by the user. If no loop is desired over a particular parameter, the fields $r e c(i$,iUpper $)$ and $r e c(i$,iStep $)$ can be omitted. On top of that there are also 'inheritance rules' (given in table 3), stating for example that M3SL defaults to MSusy if not given explicitly.

- $L$ entries indicate fields replaced by the FHLoopRecord routine while working off the loops over parameter space, i.e. these fields are updated automatically according to the current point in the loop. For example, if the Record contains

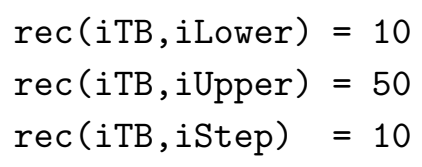

the first call to FHLoopRecord will set rec(iTB, iVar) to 10, the next to 20, etc.

\section{A.1 Fortran use}

\section{A.1.1 Declaration}

Every subroutine or function which uses a Record must first include the definitions:

\#include "FHRecord.h"

Records can then be declared with the preprocessor macro RecordDecl, which hides the declaration details. For example,

RecordDecl (rec)

declares the Record $\operatorname{rec}(i, j)$. When declaring several records, each needs its own RecordDecl statement, i.e. RecordDecl (rec1, rec2, ...) is not permissible.

\section{A.1.2 Initializing a record}

A FeynHiggs Record has to be brought into a defined state before its first use, either by clearing it with

call FHClearRecord ( $r e c)$

or by reading it from a file, which similarly overwrites any previous content:

call FHReadRecord(error, rec, "file")

if ( error .ne. 0) stop 


\begin{tabular}{|c|c|c|}
\hline Index name & Parameter & Default value \\
\hline iAlfasMZ & $\alpha_{s}\left(M_{Z}^{2}\right)$ & -1 \\
\hline $\mathrm{IMC}$ & $m_{c}$ & -1 \\
\hline iMT & $m_{t}$ & \\
\hline iMB & $m_{b}($ on-shell $)$ & -1 \\
\hline iMW & $M_{W}$ & -1 \\
\hline iMZ & $M_{Z}$ & -1 \\
\hline $\mathrm{TB}$ & $\tan \beta^{-}$ & \\
\hline MAO & $M_{A^{0}}$ & \\
\hline MHp & $M_{H^{+}}$ & \\
\hline iMsusy & $M_{\mathrm{SUSY}}$ & \\
\hline iM3SL & $M_{\tilde{L}}^{3}$ & iMSusy \\
\hline iM2SL & $M_{\tilde{L}}^{\frac{1}{2}}$ & iM3SL \\
\hline iM1SL & $M_{\tilde{\tilde{L}}}^{1}$ & iM2SL \\
\hline iM3SE & $M_{\tilde{F}}^{\frac{1}{3}}$ & iMSusy \\
\hline iM2SE & $M_{\tilde{E}}^{2}$ & iM3SE \\
\hline iM1SE & $M_{\tilde{E}}^{E}$ & iM2SE \\
\hline iM3SQ & $M_{\tilde{Q}}^{3}$ & iMSusy \\
\hline iM2SQ & $M_{\tilde{Q}}^{2}$ & iM3SQ \\
\hline iM1SQ & $M_{\tilde{O}}^{1}$ & iM2SQ \\
\hline iM3SU & $M_{\tilde{U}}^{3}$ & iMSusy \\
\hline iM2SU & $M_{\tilde{U}}^{2}$ & iM3SU \\
\hline iM1SU & $M_{\tilde{U}}^{1}$ & iM2SU \\
\hline iM3SD & $M_{\tilde{D}}^{3}$ & iMSusy \\
\hline iM2SD & $M_{\tilde{D}}^{2}$ & iM3SD \\
\hline iM1SD & $M_{\tilde{D}}^{1}$ & iM2SD \\
\hline iQtau & $Q_{\tau}$ & 0 \\
\hline iQt & $Q_{t}$ & 0 \\
\hline$i Q b$ & $Q_{b}$ & 0 \\
\hline iCKMtheta 12 & $\theta_{12}$ & -1 \\
\hline iCKMtheta23 & $\theta_{23}$ & -1 \\
\hline iCKMtheta13 & $\theta_{13}$ & -1 \\
\hline iCKMdelta13 & $\delta_{13}$ & -1 \\
\hline
\end{tabular}

\begin{tabular}{|c|c|c|}
\hline Index name & Parameter & Default value \\
\hline iM1 & $M_{1}$ & 0 \\
\hline iM2 & $M_{2}$ & \\
\hline iM3 & $M_{3}$ & \\
\hline$i A \bar{t}$ & ${ }^{---}$ & \\
\hline iAc & $A_{c}$ & iAt \\
\hline$i \mathrm{Au}$ & $A_{u}$ & iAc \\
\hline$i \mathrm{Ab}$ & $A_{b}$ & iAt \\
\hline iAs & $A_{s}$ & $i \mathrm{Ab}$ \\
\hline iAd & $A_{d}$ & iAs \\
\hline iAtau & $A_{\tau}$ & $i \mathrm{Ab}$ \\
\hline iAmu & $A_{\mu}$ & iAtau \\
\hline iAe & $A_{e}$ & iAmu \\
\hline ideltaLLuc & $\bar{\delta}_{u c}^{L L^{-}}$ & 0 \\
\hline ideltaLRuc & $\delta_{u c}^{L R}$ & 0 \\
\hline ideltaRLuc & $\delta_{u c}^{R L}$ & 0 \\
\hline ideltaRRuc & $\delta_{u c}^{R R}$ & 0 \\
\hline ideltaLLct & $\delta_{c t}^{L L}$ & 0 \\
\hline ideltaLRct & $\delta_{c t}^{L R}$ & 0 \\
\hline ideltaRLct & $\delta_{c t}^{R L}$ & 0 \\
\hline ideltaRRct & $\delta_{c t}^{R R}$ & 0 \\
\hline ideltaLLut & $\delta_{u t}^{L L}$ & 0 \\
\hline ideltaLRut & $\delta_{u t}^{L R}$ & 0 \\
\hline ideltaRLut & $\delta_{u t}^{R L}$ & 0 \\
\hline ideltaRRut & $\delta_{u t}^{R R}$ & 0 \\
\hline ideltaLLds & $\delta_{d s}^{L L}$ & 0 \\
\hline ideltaLRds & $\delta_{d s}^{L R}$ & 0 \\
\hline ideltaRLds & $\delta_{d s}^{R S L}$ & 0 \\
\hline ideltaRRds & $\delta_{d s}^{d s}$ & 0 \\
\hline ideltaLLsb & $\begin{array}{l}d s \\
\delta_{s b}^{L L}\end{array}$ & 0 \\
\hline ideltaLRsb & $\delta_{s b}^{s b}$ & 0 \\
\hline ideltaRLsb & $\delta_{s b}^{s D}$ & 0 \\
\hline ideltaRRsb & $\delta_{s b}^{s D}$ & 0 \\
\hline ideltaLLdb & $\delta_{d b}^{s b}$ & 0 \\
\hline ideltaLRdb & $\delta_{d b}^{L o} R$ & 0 \\
\hline ideltaRLdb & $\delta_{d b}^{R L}$ & 0 \\
\hline ideltaRRdb & $\delta_{d b}^{R R}$ & 0 \\
\hline
\end{tabular}

Table 3: The parameter index names of a FeynHiggs Record. Indices of real parameters are listed in the left, of complex ones in the right column. Complex quantities, e.g. $A_{t}$, can be accessed either through $\operatorname{Re}$ (iAt) and $\operatorname{Im}(i A t)$, or Abs (iAt) and $\operatorname{Arg}$ (iAt), with iAt alone as a synonym for $\operatorname{Re}$ (iAt). In cases where both Re/Im and Abs/Arg are given, the latter take precendence. Please consult the FeynHiggs(1) manual page for more details.

where file is the name of a parameter file in FeynHiggs' native format.

Fields can be set or read out using ordinary Fortran array access, e.g. 


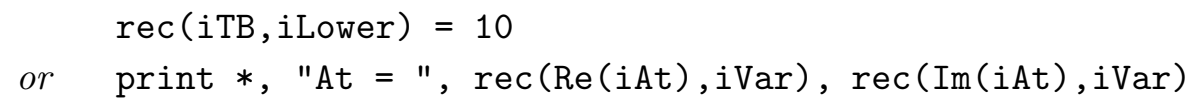

The 'current value' field (iVar) should not be set explicitly, as it is updated automatically by FHLoopRecord.

\section{A.1.3 Looping over a record/setting the FeynHiggs input}

The loops over parameters contained in a Record are worked off through calls to FHLoopRecord, which update the Record's 'current value' fields (iVar). FHLoopRecord is thus usually invoked in the context of a looping construct, such as

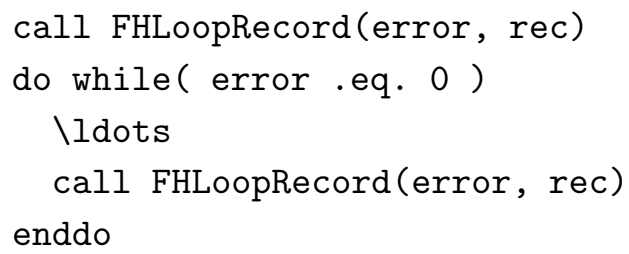

The subroutine FHSetRecord can be used to set the 'current value' fields (iVar) as input parameters for FeynHiggs. This works effectively as a combination of FHSetPara, FHSetCKM, and FHSetNMFV, except that the parameters are taken from the Record. In a typical application the above loop would be extended to

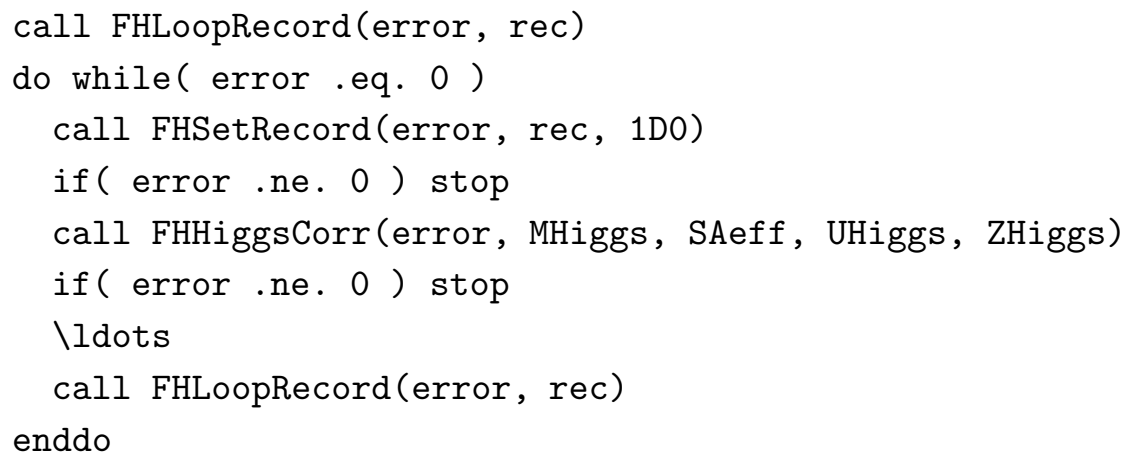

The third argument in FHSetRecord is the same scale factor which appears in FHSetPara and which determines the renormalization scale as a multiple of the top mass.

\section{A.1.4 Associating a record with a table}

The FeynHiggs Record allows one to interpolate parameters from a data table. The table is interpolated in two user-selectable variables which can be chosen identical if interpolation in only one variable is desired.

The table first needs to be loaded into internal storage. At the moment FeynHiggs has a static allocation for one table of at most 2400 lines. This allows the complete implementation in Fortran and seems sufficient for all present applications. The table's format is rather straightforward: the first line contains the column names (same identifiers as in the FeynHiggs input file), followed by the data rows. All items are separated by whitespace. 
Loading the table can either be done through the input file and is thus automatically performed in FHReadRecord. To this end one has to add a line

table file var1 var2

to the parameter file. For example, "table mytable TB MAO" reads the file mytable into memory and sets TB and MAO as input variables for the interpolation. The table must obviously contain columns for the input variables.

It is also possible to integrate the table file into the parameter file. The table statement then takes the form

table - var1 var2

and must be the last statement in the parameter file, followed immediately by the table data.

Alternately, the table is loaded by

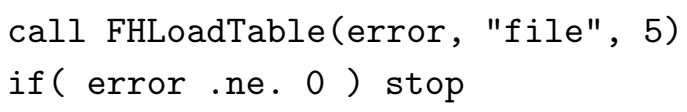

The table is read from file, unless that equals "-", in which case the table is read from the Fortran unit given in the third argument (unit 5 is Fortran's equivalent of stdin and hence a good default argument here).

The table is associated with the record through

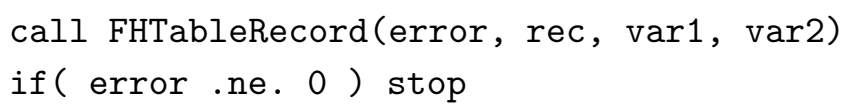

where var1 and var2 are the indices of the input variables, e.g. iTB and iMA0. To translate parameter names (strings) into indices, one can use the FHRecordIndex subroutine, as in:

call FHRecordIndex(index, name)

\section{A.2 Mathematica use}

Using FeynHiggs Records in Mathematica is for the larger part very similar to doing so in Fortran. The main difference is that one does not have to declare a Record. Rather, both initialization routines 'create' the Record:

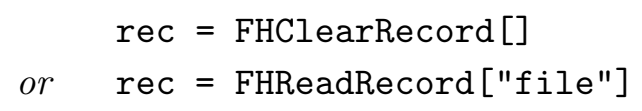

The Record is represented as an FHRecord object in Mathematica. Access to fields is very similar to the Fortran case, e.g.

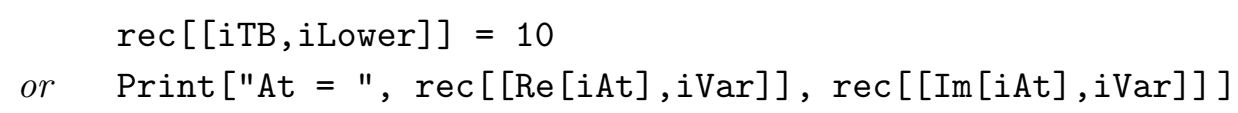


So is the use of FHLoopRecord, except that the updated Record is returned, rather than modified in situ. In other words, FHLoopRecord returns an FHRecord as long as the loop continues. The loop would thus look like

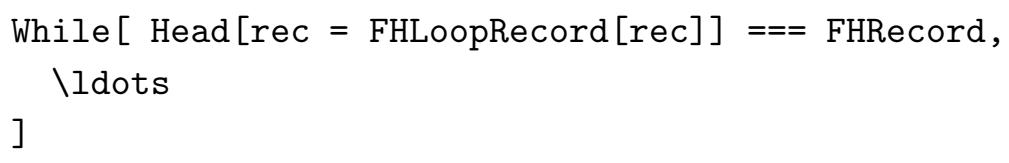

The other routines are used straightforwardly, for example:

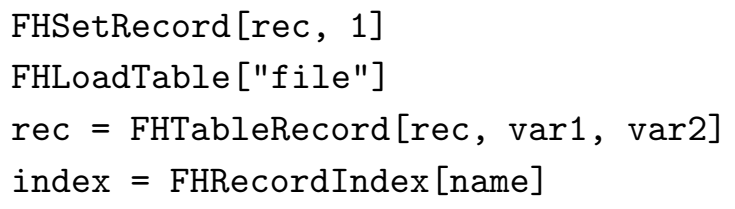

\section{A.3 Examples}

\section{A.3.1 Command-line mode with parameter table}

In the simplest case, a Parameter table can be processed through an input file with a table statement:
MAO 203
TB 5.7

table file.dat MAO TB

The Parameter table is read from file.dat in a format like

$\begin{array}{llllll}\text { MT } & \text { MSusy } & \text { MAO } & \text { TB } & \text { At } & \text { MUE \ldots } \\ 171.4 & 500 & 200 & 5 & 1000 & 761 \\ 171.4 & 500 & 210 & 5 & 1000 & 753 \\ \text { Ildots } & & & & \\ 171.4 \quad 500 & 200 & 6 & 1000 & 742 \\ 171.4 \quad 500 & 210 & 6 & 1000 & 735 \\ \text { \ldots } & & & & & \end{array}$

Alternately, the table can be integrated into the parameter file, as in

$\begin{array}{llllll}\text { MAO } & 203 & & & & \\ \text { TB } & 5.7 & & & & \\ \text { table } & - \text { MAO } & \text { TB } & & & \\ \text { MT } & \text { MSusy } & \text { MAO } & \text { TB } & \text { At } & \text { MUE \ldots } \\ 171.4 & 500 & 200 & 5 & 1000 & 761 \\ 171.4 & 500 & 210 & 5 & 1000 & 753 \\ \text { Ildots } & & & & \\ 171.4 & 500 & 200 & 6 & 1000 & 742 \\ 171.4 & 500 & 210 & 6 & 1000 & 735 \\ \text { Ildots } & & & & \end{array}$


This minimal setup assumes that all parameters are contained in the table. More generally, the ones not contained in the table have to be given in the parameter file. The interpolation for the parameters given (here MAO and TB) is performed automatically by FeynHiggs.

\section{A.3.2 Using a record with table in Fortran}

In Fortran, the same example might be coded as

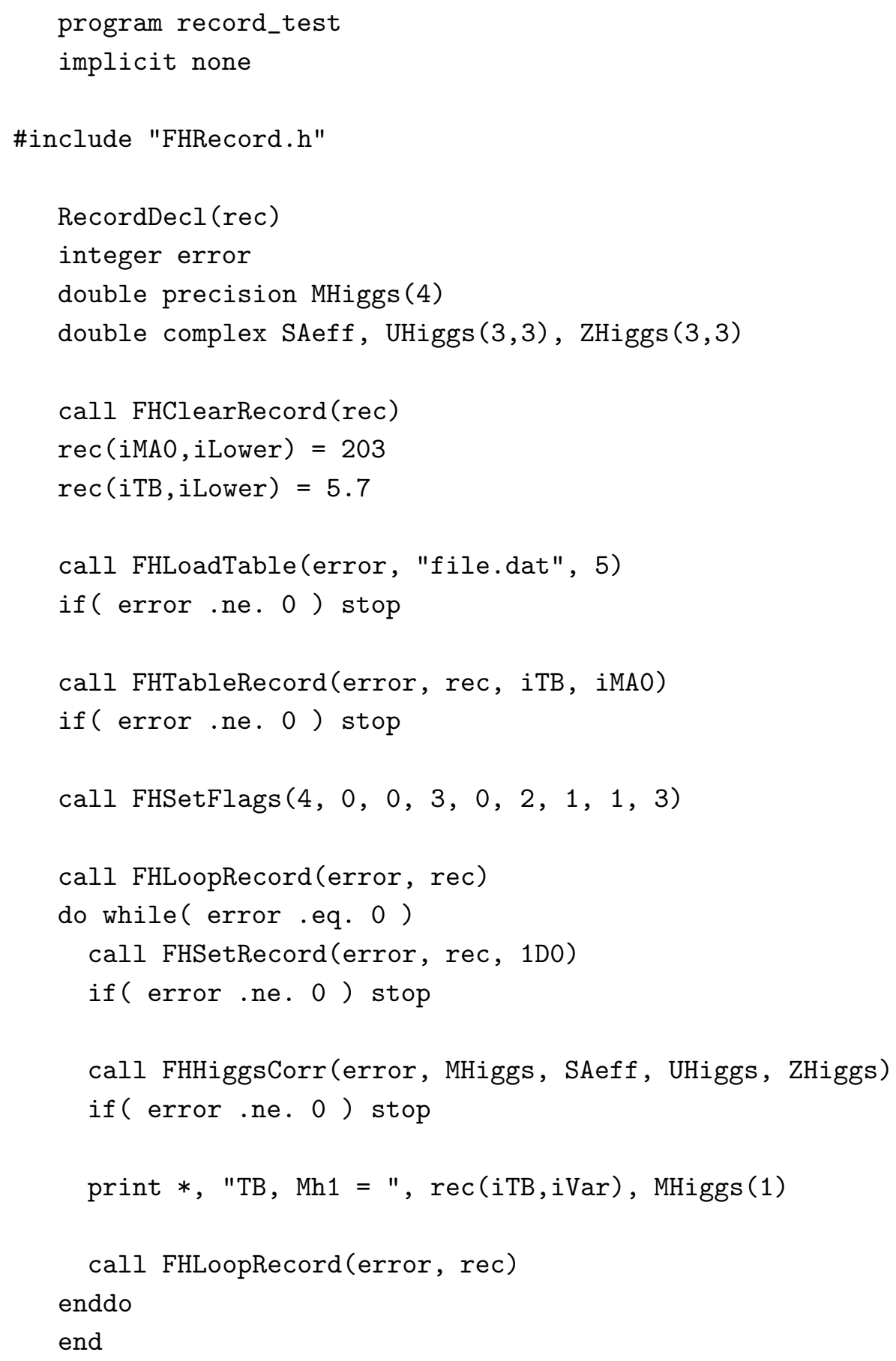




\section{A.3.3 Using a record with table in Mathematica}

In Mathematica, the structure and syntax is very similar to Fortran (mainly round brackets have to be converted into square ones):

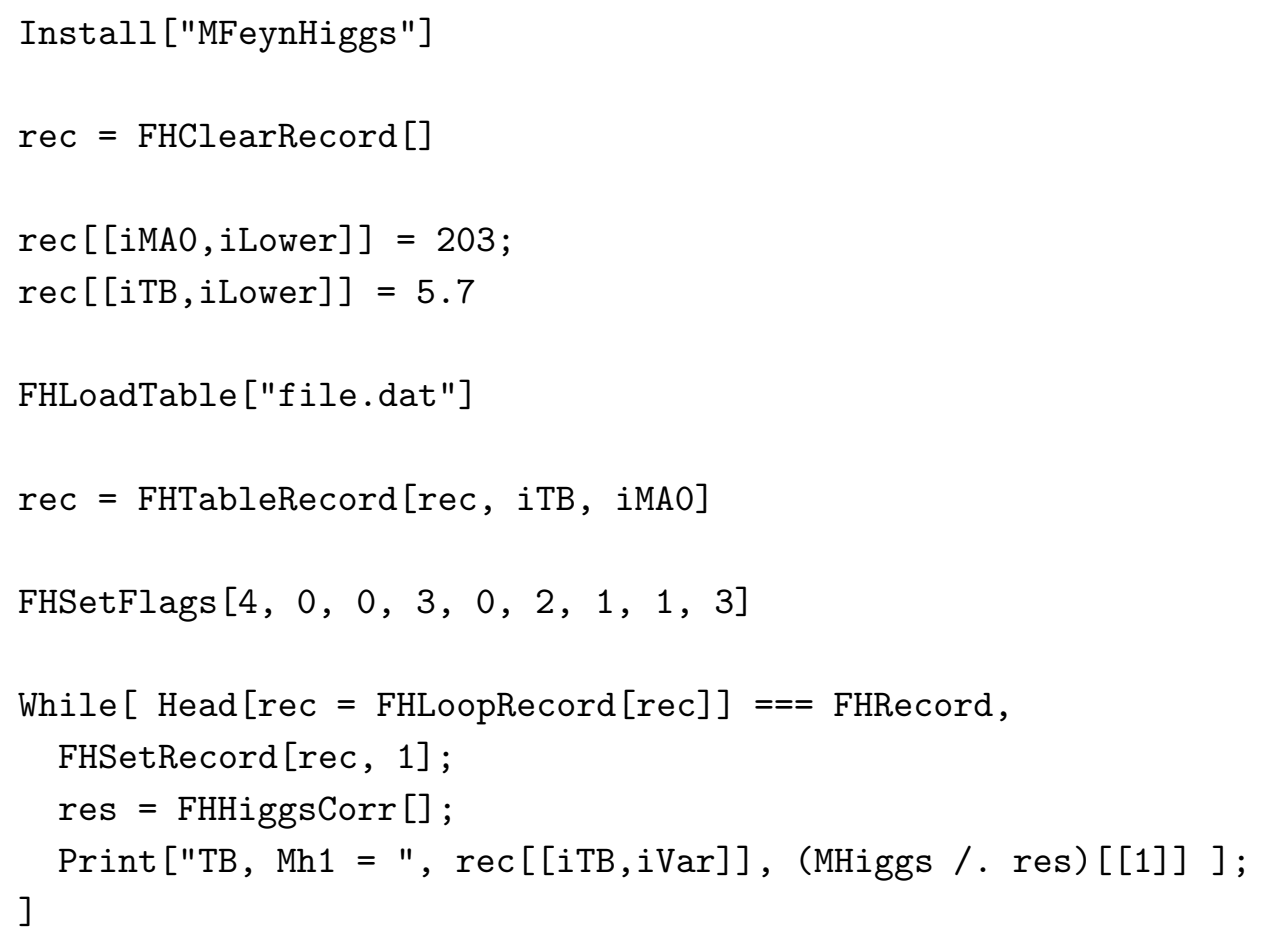

\section{References}

[1] H.P. Nilles, Supersymmetry, supergravity and particle physics, Phys. Rept. 110 (1984)1.

[2] H.E. Haber and G.L. Kane, The search for supersymmetry: probing physics beyond the standard model, Phys. Rept. 117 (1985) 75;

E. Fermi, Trends to a theory of beta radiation (in italian)., Nuovo Cim. 11 (1934) 1.

[3] D0 collaboration, G. Bernardi et al., Combined D0 and CDF upper limits on standard-model Higgs-boson production, hep-ex/0612044.

[4] CDF collaboration, CDF note 8774, CDF note 8742, CDF note 8442 and CDF note 8390, see: http://www-cdf.fnal.gov/physics/new/hdg/hdg.htm];

CDF collaboration, A. Abulencia et al., Search for a neutral Higgs boson decaying to a $W$ boson pair in p p collisions at $\sqrt{s}=1.96 \mathrm{TeV}$, Phys. Rev. Lett. $97(2006) 081802$ hep-ex/0605124.

[5] D0 collaboration, D0 Note 5380-CONF, D0 Note 5365-CONF, D0 Note 535\%-CONF, D0 Note 5353-CONF and DO Note 5275-CONF, see: http://www-d0.fnal.gov/Run2Physics/WWW/results/higgs.htm; D0 collaboration, V.M. Abazov et al., Search for associated Higgs boson production $W H \rightarrow$ $W W W * \rightarrow \ell^{ \pm} \nu \ell^{ \pm \prime} \nu^{\prime}+x$ in $p \bar{p}$ collisions at $\sqrt{s}=1.96 \mathrm{TeV}$, Phys. Rev. Lett. $97(2006)$ 151804 hep-ex/0607032;

D0 collaboration, V.M. Abazov et al., Search for the standard model Higgs boson in the $p \bar{p} \rightarrow$ $Z H \rightarrow \nu \bar{\nu} b \bar{b}$ channel, Phys. Rev. Lett. 97 (2006) 161803 hep-ex/0607022]. 
[6] CDF collaboration, A. Abulencia et al., Search for neutral MSSM Higgs bosons decaying to tau pairs in $p \bar{p}$ collisions at $\sqrt{s}=1.96$ TeV, Phys. Rev. Lett. 96 (2006) 011802 hep-ex/0508051.

[7] D0 collaboration, V.M. Abazov et al., Search for neutral Higgs bosons decaying to $\tau$ pairs in $p \bar{p}$ collisions at $\sqrt{s}=1.96$ TeV, Phys. Rev. Lett. 97 (2006) 121802 hep-ex/0605009.

[8] CDF collaboration, A. Abulencia et al., Search for charged Higgs bosons from top quark decays in $p \bar{p}$ collisions at $\sqrt{s}=1.96$ TeV, Phys. Rev. Lett. 96 (2006) 042003 hep-ex/0510065.

[9] CDF collaboration, CDF note 8676, see http://www-cdf.fnal.gov/ aa/mssm_htt_1fb/note/cdf8676.pdf.

[10] D0 collaboration, Do Note 5331-CONF, see http://www-d0.fnal.gov/cgi-bin/d0note?5331.

[11] ATLAS collaboration, Detector and physics performance technical design report, CERN-LHCC-99-15 (1999), see http://atlasinfo.cern.ch/Atlas/GROUPS/PHYSICS/TDR/access.htm.

[12] M. Schumacher, Investigation of the discovery potential for Higgs bosons of the minimal supersymmetric extension of the standard model (MSSM) with ATLAS, hep-ph/0410112.

[13] S. Abdullin et al., Summary of the CMS potential for the Higgs boson discovery, Eur. Phys. J. C 39 (2005) 41.

[14] CMS collaboration, Physics technical design report. Volume 2., CERN-LHCC-2006-021, see: http://cmsdoc.cern.ch/cms/cpt/tdr/.

[15] ECFA/DESY LC Physics Working Group collaboration, J.A. Aguilar-Saavedra et al., Tesla technical design report part III: physics at an $e^{+} e^{-}$linear collider, hep-ph/0106315; see http://tesla.desy.de/tdr/.

[16] American Linear Collider Working Group collaboration, T. Abe et al., Linear collider physics resource book for snowmass 2001. 1: introduction, hep-ex/0106055.

[17] ACFA Linear Collider Working Group collaboration, K. Abe et al., Particle physics experiments at $J L C$, hep-ph/0109166.

[18] S. Heinemeyer et al., Toward high precision Higgs-boson measurements at the international linear $e^{+} e^{-}$collider, eConf C0508141 (2005) ALCPG0214 hep-ph/0511332.

[19] A. Djouadi, The anatomy of electro-weak symmetry breaking. II: the Higgs bosons in the minimal supersymmetric model, hep-ph/0503173.

[20] K. Desch, E. Gross, S. Heinemeyer, G. Weiglein and L. Zivkovic, LHC/LC interplay in the MSSM Higgs sector, JHEP 09 (2004) 062 hep-ph/0406322].

[21] J.R. Ellis, S. Heinemeyer, K.A. Olive and G. Weiglein, Precision analysis of the lightest MSSM Higgs boson at future colliders, JHEP 01 (2003) 006 hep-ph/0211206.

[22] A. Dedes, S. Heinemeyer, S. Su and G. Weiglein, The lightest Higgs boson of mSUGRA, $m G M S B$ and $m A M S B$ at present and future colliders: observability and precision analyses, Nucl. Phys. B 674 (2003) 271 hep-ph/0302174.

[23] CLIC Physics Working Group collaboration, E. Accomando et al., Physics at the clic multi-TeV linear collider, hep-ph/0412251. 
[24] S. Heinemeyer, W. Hollik and G. Weiglein, Electroweak precision observables in the minimal supersymmetric standard model, Phys. Rept. 425 (2006) 265 hep-ph/0412214.

[25] J.R. Ellis, S. Heinemeyer, K.A. Olive, A.M. Weber and G. Weiglein, The supersymmetric parameter space in light of B-physics observables and electroweak precision data, JHEP 08 (2007) 083 arXiv:0706.0652.

[26] CDMS collaboration, D.S. Akerib et al., Limits on spin-independent wimp nucleon interactions from the two-tower run of the cryogenic dark matter search, Phys. Rev. Lett. 96 (2006) 011302 astro-ph/0509259.

[27] XENON collaboration, J. Angle et al., First results from the XENON10 dark matter experiment at the Gran Sasso national laboratory, arXiv:0706.0039.

[28] A. Djouadi and Y. Mambrini, The Higgs intense-coupling regime in constrained SUSY models and its astrophysical implications, JHEP 12 (2006) 001 hep-ph/0609234.

[29] M.S. Carena, D. Hooper and P. Skands, Implications of direct dark matter searches for MSSM Higgs searches at the Tevatron, Phys. Rev. Lett. 97 (2006) 051801 hep-ph/0603180.

[30] J.R. Ellis, S. Heinemeyer, K.A. Olive and G. Weiglein, Light heavy MSSM Higgs bosons at large $\tan \beta$, Phys. Lett. B 653 (2007) 292 arXiv:0706.0977.

[31] M.S. Carena, S. Heinemeyer, C.E.M. Wagner and G. Weiglein, Suggestions for improved benchmark scenarios for Higgs-boson searches at LEP2, hep-ph/9912223.

[32] M.S. Carena, S. Heinemeyer, C.E.M. Wagner and G. Weiglein, Suggestions for benchmark scenarios for MSSM Higgs boson searches at hadron colliders, Eur. Phys. J. C 26 (2003) 601 hep-ph/0202167.

[33] M.S. Carena, S. Heinemeyer, C.E.M. Wagner and G. Weiglein, MSSM Higgs boson searches at the Tevatron and the LHC: impact of different benchmark scenarios, Eur. Phys. J. C 45 (2006) 797 hep-ph/0511023.

[34] M.S. Carena, J.R. Ellis, A. Pilaftsis and C.E.M. Wagner, CP-violating MSSM Higgs bosons in the light of LEP2, Phys. Lett. B 495 (2000) 155 hep-ph/0009212].

[35] M. Battaglia et al., Proposed post-LEP benchmarks for supersymmetry, Eur. Phys. J. C 22 (2001) 535 hep-ph/0106204.

[36] B.C. Allanach et al., The snowmass points and slopes: benchmarks for SUSY searches, hep-ph/0202233.

[37] M. Battaglia et al., Updated post-WMAP benchmarks for supersymmetry, Eur. Phys. J. C 33 (2004) 273 hep-ph/0306219.

[38] A. De Roeck et al., Supersymmetric benchmarks with non-universal scalar masses or gravitino dark matter, Eur. Phys. J. C 49 (2007) 1041 hep-ph/0508198.

[39] S. Heinemeyer, W. Hollik and G. Weiglein, Constraints on $\tan \beta$ in the MSSM from the upper bound on the mass of the lightest Higgs boson, JHEP 06 (2000) 009 hep-ph/9909540.

[40] J.R. Ellis, K.A. Olive and Y. Santoso, The MSSM parameter space with non-universal Higgs masses, Phys. Lett. B 539 (2002) 107 hep-ph/0204192.

[41] J.R. Ellis, T. Falk, K.A. Olive and Y. Santoso, Exploration of the MSSM with non-universal Higgs masses, Nucl. Phys. B 652 (2003) 259 hep-ph/0210205. 
[42] M. Olechowski and S. Pokorski, Electroweak symmetry breaking with nonuniversal scalar soft terms and large $\tan \beta$ solutions, Phys. Lett. B 344 (1995) 201 hep-ph/9407404;

V. Berezinsky et al., Neutralino dark matter in supersymmetric models with nonuniversal scalar mass terms, Astropart. Phys. 5 (1996) 1 hep-ph/9508249];

M. Drees, M.M. Nojiri, D.P. Roy and Y. Yamada, Light higgsino dark matter, Phys. Rev. D 56 (1997) 276 [Erratum ibid. D 64 (1997) 039901] hep-ph/9701219];

M. Drees et al., Scrutinizing LSP dark matter at the LHC, Phys. Rev. D 63 (2001) 035008 hep-ph/0007202;

P. Nath and R. Arnowitt, Non-universal soft SUSY breaking and dark matter, Phys. Rev. D 56 (1997) 2820 hep-ph/9701301;

J.R. Ellis, T. Falk, G. Ganis, K.A. Olive and M. Schmitt, Charginos and neutralinos in the light of radiative corrections: sealing the fate of higgsino dark matter, Phys. Rev. D 58 (1998) 095002 hep-ph/9801445;

J.R. Ellis, T. Falk, G. Ganis and K.A. Olive, Supersymmetric dark matter in the light of LEP and the Tevatron collider, Phys. Rev. D 62 (2000) 075010 hep-ph/0004169;

A. Bottino, F. Donato, N. Fornengo and S. Scopel, Probing the supersymmetric parameter space by wimp direct detection, Phys. Rev. D 63 (2001) 125003 hep-ph/0010203;

S. Profumo, Neutralino dark matter, B- $\tau$ Yukawa unification and non-universal sfermion masses, Phys. Rev. D 68 (2003) 015006 hep-ph/0304071;

D.G. Cerdeno and C. Muñoz, Neutralino dark matter in supergravity theories with nonuniversal scalar and gaugino masses, JHEP 10 (2004) 015 hep-ph/0405057;

H. Baer, A. Mustafayev, S. Profumo, A. Belyaev and X. Tata, Direct, indirect and collider detection of neutralino dark matter in SUSY models with non-universal Higgs masses, JHEP 07 (2005) 065 hep-ph/0504001.

[43] WMAP collaboration, C.L. Bennett et al., First year Wilkinson Microwave Anisotropy Probe (WMAP) observations: preliminary maps and basic results, Astrophys. J. Suppl. 148 (2003) 1 astro-ph/0302207;

WMAP collaboration, D.N. Spergel et al., First year Wilkinson Microwave Anisotropy Probe (WMAP) observations: determination of cosmological parameters, Astrophys. J. Suppl. 148 (2003) 175 astro-ph/0302209;

WMAP collaboration, D.N. Spergel et al., Wilkinson Microwave Anisotropy Probe (WMAP) three year results: implications for cosmology, Astrophys. J. Suppl. 170 (2007) 377 astro-ph/0603449.

[44] J.R. Ellis, K.A. Olive, Y. Santoso and V.C. Spanos, Supersymmetric dark matter in light of WMAP, Phys. Lett. B 565 (2003) 176 hep-ph/0303043.

[45] C. Muñoz, Dark matter detection in the light of recent experimental results, Int. J. Mod. Phys. A 19 (2004) 3093 hep-ph/0309346;

R. Arnowitt, B. Dutta and B. Hu, Dark matter, muon $g-2$ and other SUSY constraints, hep-ph/0310103;

H. Baer and C. Balázs, $\chi^{2}$ analysis of the minimal supergravity model including WMAP, $g(\mu)-2$ and $b \rightarrow s \gamma$ constraints, JCAP 05 (2003) 006 hep-ph/0303114;

A.B. Lahanas and D.V. Nanopoulos, Wmaping out supersymmetric dark matter and phenomenology, Phys. Lett. B 568 (2003) 55 hep-ph/0303130;

U. Chattopadhyay, A. Corsetti and P. Nath, Wmap constraints, SUSY dark matter and implications for the direct detection of SUSY, Phys. Rev. D 68 (2003) 035005

hep-ph/0303201. 
[46] H. Goldberg, Constraint on the photino mass from cosmology, Phys. Rev. Lett. 50 (1983) 1419 ;

J.R. Ellis, J.S. Hagelin, D.V. Nanopoulos, K.A. Olive and M. Srednicki, Supersymmetric relics from the Big Bang, Nucl. Phys. B 238 (1984) 453.

[47] J.R. Ellis, K.A. Olive, Y. Santoso and V.C. Spanos, On $B_{s} \rightarrow \mu^{+} \mu^{-}$and cold dark matter scattering in the MSSM with non-universal Higgs masses, JHEP 05 (2006) 063 hep-ph/0603136.

[48] J.R. Ellis, S. Heinemeyer, K.A. Olive and G. Weiglein, Indirect sensitivities to the scale of supersymmetry, JHEP 02 (2005) 013 hep-ph/0411216.

[49] J.R. Ellis, S. Heinemeyer, K.A. Olive and G. Weiglein, Phenomenological indications of the scale of supersymmetry, JHEP 05 (2006) 005 hep-ph/0602220.

[50] J.R. Ellis, K.A. Olive, Y. Santoso and V.C. Spanos, Likelihood analysis of the cmssm parameter space, Phys. Rev. D 69 (2004) 095004 hep-ph/0310356;

B.C. Allanach and C.G. Lester, Multi-dimensional mSUGRA likelihood maps, Phys. Rev. D 73 (2006) 015013 hep-ph/0507283;

B.C. Allanach, Naturalness priors and fits to the constrained minimal supersymmetric standard model, Phys. Lett. B 635 (2006) 123 hep-ph/0601089;

R.R. de Austri, R. Trotta and L. Roszkowski, A Markov chain Monte Carlo analysis of the CMSSM, JHEP 05 (2006) 002 hep-ph/0602028;

L. Roszkowski, R.R. de Austri and R. Trotta, On the detectability of the cmssm light Higgs boson at the Tevatron, JHEP 04 (2007) 084 hep-ph/0611173; Implications for the constrained MSSM from a new prediction for $B \rightarrow S \gamma$, JHEP 07 (2007) 075 arXiv:0705.2012;

B.C. Allanach, C.G. Lester and A.M. Weber, The dark side of mSUGRA, JHEP 12 (2006) 065 hep-ph/0609295;

B.C. Allanach, K. Cranmer, C.G. Lester and A.M. Weber, Natural priors, CMSSM fits and LHC weather forecasts, JHEP 08 (2007) 023 arXiv:0705.0487;

O. Buchmueller et al., Prediction for the lightest Higgs boson mass in the CMSSM using indirect experimental constraints, arXiv:0707.3447.

[51] J.R. Ellis, K.A. Olive, Y. Santoso and V.C. Spanos, Update on the direct detection of supersymmetric dark matter, Phys. Rev. D 71 (2005) 095007 hep-ph/0502001.

[52] ALEPH collaboration, S. Schael et al., Search for neutral MSSM Higgs bosons at LEP, Eur. Phys. J. C 47 (2006) 547 hep-ex/0602042.

[53] LEP Working Group for Higgs boson searches collaboration, R. Barate et al., Search for the standard model Higgs boson at LEP, Phys. Lett. B 565 (2003) 61 hep-ex/0306033.

[54] G. Degrassi, S. Heinemeyer, W. Hollik, P. Slavich and G. Weiglein, Towards high-precision predictions for the MSSM Higgs sector, Eur. Phys. J. C 28 (2003) 133 hep-ph/0212020.

[55] S. Heinemeyer, W. Hollik and G. Weiglein, Feynhiggs: a program for the calculation of the masses of the neutral CP-even Higgs bosons in the MSSM, Comput. Phys. Commun. 124 (2000) 76 hep-ph/9812320. The code is accessible via http://www.feynhiggs.de.

[56] S. Heinemeyer, W. Hollik and G. Weiglein, The masses of the neutral CP-even Higgs bosons in the MSSM: accurate analysis at the two-loop level, Eur. Phys. J. C 9 (1999) 343 hep-ph/9812472. 
[57] M. Frank et al., The Higgs boson masses and mixings of the complex MSSM in the Feynman-diagrammatic approach, JHEP 02 (2007) 047 hep-ph/0611326.

[58] T. Moroi, The muon anomalous magnetic dipole moment in the minimal supersymmetric standard model, Phys. Rev. D 53 (1996) 6565 [Erratum ibid. 56 (1997) 4424] hep-ph/9512396.

[59] G. Degrassi and G.F. Giudice, QED logarithms in the electroweak corrections to the muon anomalous magnetic moment, Phys. Rev. D 58 (1998) 053007 hep-ph/9803384.

[60] S. Heinemeyer, D. Stöckinger and G. Weiglein, Two-loop SUSY corrections to the anomalous magnetic moment of the muon, Nucl. Phys. B 690 (2004) 62 hep-ph/0312264.

[61] S. Heinemeyer, D. Stöckinger and G. Weiglein, Electroweak and supersymmetric two-loop corrections to $(g-2)(\mu)$, Nucl. Phys. B 699 (2004) 103 hep-ph/0405255.

[62] Muon $g-2$ collaboration, G.W. Bennett et al., Measurement of the negative muon anomalous magnetic moment to 0.7 ppm, Phys. Rev. Lett. 92 (2004) 161802 hep-ex/0401008.

[63] Muon G-2 collaboration, G.W. Bennett et al., Final report of the muon E821 anomalous magnetic moment measurement at BNL, Phys. Rev. D 73 (2006) 072003 hep-ex/0602035.

[64] M. Davier, The hadronic contribution to $(g-2)(\mu)$, Nucl. Phys. 169 (Proc. Suppl.) (2007) 288 hep-ph/0701163.

[65] S. Eidelman, talk given at the ICHEP06, Moscow, July (2006), see: http://ichep06.jinr.ru/reports/333_6s1_9p30_Eidelman.pdf.

[66] K. Hagiwara, A.D. Martin, D. Nomura and T. Teubner, Improved predictions for $g-2$ of the muon and $\alpha_{\mathrm{QED}}\left(M_{Z}^{2}\right)$, Phys. Lett. B 649 (2007) 173 hep-ph/0611102.

[67] J.P. Miller, E. de Rafael and B.L. Roberts, Muon g - 2: review of theory and experiment, Rev. Mod. Phys. 70 (2007) 795 hep-ph/0703049.

[68] F. Jegerlehner, Essentials of the muon g - 2, Acta Phys. Polon. B38 (2007) 3021 hep-ph/0703125.

[69] T. Wyatt, Plenary talk at the European Physical Society HEP Conference, Manchester, July (2007), based on: KLOE collaboration, F. Ambrosino et al., Kloe extraction of $a_{\mu}^{\pi \pi}$ in the mass range [0.35, 0.95] $\mathrm{GeV}^{2}$, arXiv:0707.4078.

[70] CDF collaboration, see: http://www-cdf.fnal.gov/physics/projections/.

[71] H. Baer, M. Bisset, X. Tata and J. Woodside, Supercollider signals from gluino and squark decays to Higgs bosons, Phys. Rev. D 46 (1992) 303;

H. Baer, C.-H. Chen, F. Paige and X. Tata, Signals for minimal supergravity at the CERN large hadron collider: multi-jet plus missing energy channel, Phys. Rev. D 52 (1995) 2746 hep-ph/9503271;

A. Datta, A. Djouadi, M. Guchait and F. Moortgat, Detection of MSSM Higgs bosons from supersymmetric particle cascade decays at the LHC, Nucl. Phys. B 681 (2004) 31 hep-ph/0303095.

[72] A. Nikitenko, private communication. 
[73] J.F. Gunion and H.E. Haber, Higgs bosons in supersymmetric models. 3. decays into neutralinos and charginos, Nucl. Phys. B 307 (1988) 445 [Erratum ibid. 402 (1993) 569]; H. Baer, M. Bisset, D. Dicus, C. Kao and X. Tata, The search for Higgs bosons of minimal supersymmetry: impact of supersymmetric decay modes, Phys. Rev. D 47 (1993) 1062; H. Baer, M. Bisset, C. Kao and X. Tata, Detecting Higgs boson decays to neutralinos at hadron supercolliders, Phys. Rev. D 50 (1994) 316 hep-ph/9402265.

[74] F. Moortgat, S. Abdullin and D. Denegri, Observability of MSSM Higgs bosons via sparticle decay modes in $C M S$, hep-ph/0112046.

[75] M. Bisset, J. Li, N. Kersting, F. Moortgat and S. Moretti, Four-lepton LHC events from MSSM Higgs boson decays into neutralino and chargino pairs, arXiv:0709.1029, M. Bisset, J. Li and N. Kersting, How to detect 'decoupled' heavy supersymmetric Higgs bosons, arXiv:0709.1031.

[76] S. Gennai, A. Nikitenko and L. Wendland, Search for MSSM heavy neutral Higgs boson in $\tau \tau \rightarrow$ two jet decay mode, CMS Note 2006/126.

[77] R. Kinnunen and S. Lehti, Search for the heavy neutral MSSM Higgs bosons with the $H / A \rightarrow \tau^{+} \tau^{-}+$electron + jet decay mode, CMS Note 2006/075.

[78] A. Kalinowski, M. Konecki and D. Kotlinski, Search for MSSM heavy neutral. Higgs boson in $\tau+$ tau $\rightarrow \mu+$ jet decay mode, CMS Note 2006/105.

[79] S. Gennai et al., Search for heavy neutral MSSM Higgs bosons with CMS: reach and Higgs-mass precision, Eur. Phys. J. C 52 (2007) 383 arXiv:0704.0619.

[80] M. Dührssen et al., Extracting Higgs boson couplings from LHC data, Phys. Rev. D 70 (2004) 113009 hep-ph/0406323]; Determination of Higgs-boson couplings at the LHC, hep-ph/0407190.

[81] M. Dührssen, The determination of the Higgs boson couplings to fermions and bosons, ATL-PHYS-2003-030, see http://cdsweb.cern.ch.

[82] J. Brient, Talk at the Linear Collider Workshop, Cracow, Poland, September (2001), see http://webnt.physics.ox.ac.uk/lc/ecfadesy.

[83] T.L. Barklow, Higgs coupling measurements at a 1 TeV linear collider, hep-ph/0312268.

[84] J.R. Ellis, K.A. Olive and V.C. Spanos, On the interpretation of $B_{s} \rightarrow \mu^{+} \mu^{-}$in the CMSSM, Phys. Lett. B 624 (2005) 47 hep-ph/0504196.

[85] CDF collaboration, CDF Public Note 8176, see http://www-cdf.fnal.gov/physics/new/bottom/060316.blessed-bsmumu3A.

[86] D0 collaboration, D0 Note 5344-Conf, see http://www-d0.fnal.gov/Run2Physics/WWW/results/b.htm.

[87] K. Tollefson, Talk given at Lepton Photon 07, Daegu, Korea, August (2007), see http://chep.knu.ac.kr/lp07/htm/S4/S04_14.pdt.

[88] P. Gambino and M. Misiak, Quark mass effects in $\bar{B} \rightarrow X_{s} \gamma$, Nucl. Phys. B 611 (2001) 338 hep-ph/0104034.

[89] M. Misiak et al., The first estimate of $B\left(\bar{B} \rightarrow X_{s} \gamma\right)$ at $O\left(\alpha_{s}^{2}\right)$, Phys. Rev. Lett. $98(2007)$ 022002 hep-ph/0609232. 
[90] Heavy Flavor Averaging Group (HFAG) collaboration, E. Barberio et al., Averages of $B$-hadron properties at the end of 2005, hep-ex/0603003.

[91] G. Isidori and P. Paradisi, Hints of large $\tan \beta$ in flavour physics, Phys. Lett. B 639 (2006) 499 hep-ph/0605012.

[92] K. Ikado et al., Evidence of the purely leptonic decay $B^{-} \rightarrow \tau^{-} \overline{n u}_{\tau}$, Phys. Rev. Lett. 97 (2006) 251802 hep-ex/0604018.

[93] BABAR collaboration, B. Aubert et al., A search for $B^{+} \rightarrow \tau^{+} \nu$ recoiling against $B^{-} \rightarrow$ $D_{0} l^{-} \bar{n} u_{l} x$, hep-ex/0608019.

[94] J. Gasser, H. Leutwyler and M.E. Sainio, Sigma term update, Phys. Lett. B 253 (1991) 252; M. Knecht, Working group summary: $\pi n \sigma$ term, PiN Newslett. 15 (1999) 108 hep-ph/9912443;

M.E. Sainio, Pion nucleon sigma-term: a review, PiN Newslett. 16 (2002) 138 hep-ph/0110413.

[95] P. Skands et al., SUSY Les Houches accord: interfacing SUSY spectrum calculators, decay packages and event generators, JHEP 07 (2004) 036 hep-ph/0311123;

B.C. Allanach et al., Les Houches 'physics at TeV colliders 2005' beyond the standard model working group: summary report, hep-ph/0602198;

T. Hahn, SUSY Les Houches accord I/O made easy, hep-ph/0408283; SUSY Les Houches accord 2 I/O made easy, hep-ph/0605049. 